\title{
Spin models for orientational ordering of colloidal molecular crystals
}

\author{
Andreja Šarlah, ${ }^{1}$ Erwin Frey, ${ }^{2}$ and Thomas Franosch ${ }^{2}$ \\ ${ }^{1}$ Faculty of Mathematics and Physics, Department of Physics, Univerza v Ljubljani, Jadranska 19, SI-1000 Ljubljana, Slovenia \\ ${ }^{2}$ Arnold Sommerfeld Center for Theoretical Physics (ASC) and Center for NanoScience (CeNS), Department of Physics, \\ Ludwig-Maximilians-Universität München, Theresienstrasse 37, D-80333 München, Germany
}

(Received 28 July 2006; published 16 February 2007)

\begin{abstract}
Two-dimensional colloidal suspensions exposed to periodic external fields exhibit a variety of molecular crystalline phases. There two or more colloids assemble at lattice sites of potential minima to build new structural entities, referred to as molecules. Using the strength of the potential and the filling fraction as control parameters, a phase transition to unconventional orientationally ordered states can be induced. We introduce an approach that focuses at the discrete set of orientational states relevant for the phase ordering. The orientationally ordered states are mapped to classical spin systems. We construct effective Hamiltonians for dimeric and trimeric molecules on triangular lattices suitable for a statistical mechanics discussion. A mean-field analysis produces a rich phase behavior which is substantiated by Monte Carlo simulations.
\end{abstract}

DOI: 10.1103/PhysRevE.75.021402

PACS number(s): 82.70.Dd, 64.70.Dv

\section{INTRODUCTION}

Soft materials comprised of colloidal particles undergo phase transitions from fluid to crystalline order just as their atomic counterparts [1-4]. The intrinsic time and length scales of such suspensions offer the advantage to monitor the ordering phenomena by video microscopy on a single particle level. Furthermore, the interactions can be tailored to a certain degree by controlling the surface charge of the colloids or changing the refractive index of the particles and the solvent or by screening electrostatic interaction by the addition of salt, etc. [4-6]. Colloids thus constitute fascinating model systems to study material properties. For example, new crystalline structures without known atomic analog have been found [7] for mixtures of charged colloids.

In particular, confining geometries allow us to study phase transitions in two-dimensional systems where strong thermal fluctuations often become the relevant mechanism for the melting of order. For example, close to melting a universal relation between the elastic constants is predicted in the framework of the Kosterlitz-Thouless-Halperin-NelsonYoung theory $[8-10]$ as has been recently observed experimentally [11]. Even richer phase behavior is expected if quasi-two-dimensional colloidal systems are exposed to external potentials as can be realized by interfering laser beams [12-14], periodic pinning arrays [15], imprint or stamping techniques [16]. For example, in the case of one-dimensional troughs exotic phases such as the locked smectic phase or a floating solid have been predicted $[17,18]$ and partially observed in simulations $[19,20]$ and experiments [21].

For three or more laser beams periodic patterns with two modulation directions can be achieved [22], in particular, square and triangular lattices have been studied [22-25]. The colloidal particles then accumulate in the potential minima thus imposing a modulation of the colloid density. The competition between the attraction to the potential minima and the mutual repulsion of the particles can be conveniently controlled by adjusting the filling factor, i.e., the number of colloidal particles per potential minimum. For instance, by changing the angles of incidence the lattice constant of the periodic modulation can be varied continuously. Since the electrostatic interaction is usually screened by counterions in the solvent, the lattice spacing also sensitively determines how strongly particles interact. A second route to affect the balance between external compression and internal repulsion is by changing the intensities of the laser beams. Third, the salt concentration of the solvent is a suitable control parameter to tune the range of the repulsive interaction, i.e., the Debye screening length.

This paper is motivated by recent experimental studies on two-dimensional colloidal system exposed to a potential of a triangular lattice symmetry with a filling factor 3 [22]. The density of the colloids has been selected in a regime below the spontaneous freezing transition. A continuous increase of the strength of the laser field has induced a sequence of qualitative changes of order. At low intensities of the lasers, corresponding to a potential depth of say $V_{0} \sim k_{B} T$, the colloids exhibit the typical response of a fluid to an external modulation, i.e., a small periodic component is superimposed to the homogeneous background density. Enhancing the strength of the laser potential by an order of magnitude the response becomes highly nonlinear; groups of three colloidal particles cluster in the close vicinity of each potential minimum. However, a significant number of defects - groups of two or four colloidal particles-is still present in the system. Since the filling factor has been adjusted to 3 , the number of four-groups equals the one of two-groups. In this regime the barriers separating minima become high and correspondingly single-particle diffusion is strongly suppressed [23], similar to a solid. Although no thermodynamic phase transition has occurred the system is referred to as a molecular crystal. A further increase of the external potential results in a freezeout of the defects, i.e., each lattice site is occupied by precisely three colloids. The confinement is rather strong, balanced only by the mutual electrostatic repulsion, leading to triangular arrangements of the three-groups aligned with the lattice. Interestingly, the majority of three-groups is oriented in the same way with a small number of groups adopting a mirrored configuration, see Fig. 1. Using a suitable order parameter, orientational correlations demonstrate the longrange orientational order. At still higher laser potentials the 
(a)

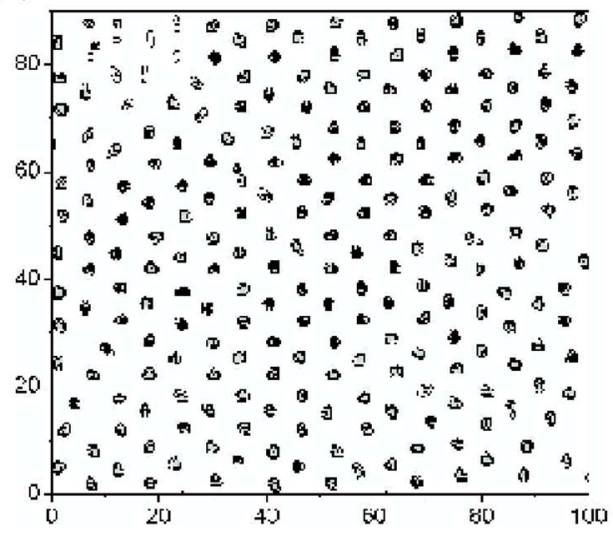

(c)



(b)

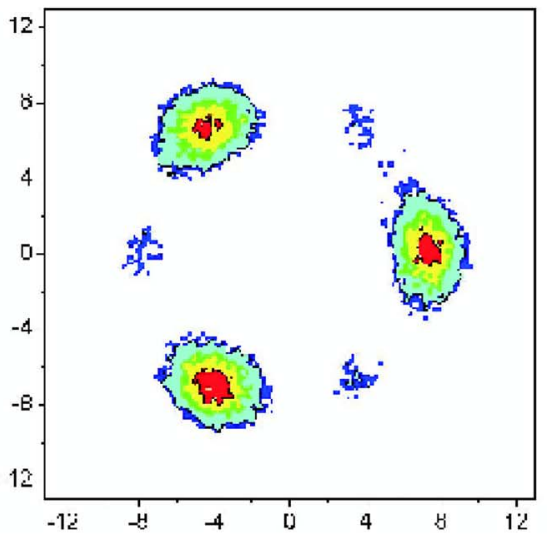

(d)



FIG. 1. (Color online) Contour plots of the lateral density distribution (a) and (c) and the averaged local particle density (b) and (d) for different light potentials: (a), (b) $V_{0}=60 k_{B} T$ and (c), (d) $V_{0}=110 k_{B} T$. In the first case the majority of three-groups of colloids is oriented in the same way, whereas in the latter case the orientation of the triangles is uncorrelated at large distances. At some intermediate $V_{0}$ a phase transition occurs. The horizontal and vertical axes are $x$ and $y$, respectively. All units are in $\mu \mathrm{m}$. Figure is reproduced from a part of Fig. 2 of Brunner and Bechinger [22]. orientation of the triangles is uncorrelated at large distances, indicating a phase transition to an orientationally disordered phase. In Ref. [22] this subsequent melting has been referred to as reentrant transition, see Fig. 1.

The possibility to induce phase transitions by periodic laser patterns has stimulated theoretical studies in twodimensional colloidal systems. Reichhardt and Olson have performed extensive Langevin simulations and have identified ground states for a series of filling factors on square and triangular lattices [23]. In particular, they have also observed the orientational ordered state corresponding to the experiment discussed above. For noninteger filling factors the molecular crystals are composed of supercells, i.e., several types of groups of colloids are distributed periodically on the optical lattice [20]. By lumping particles to rigid molecules, explicit minimizations of intermolecular interactions in the presence of the optical potential have revealed several ordered ground states [24]. Furthermore, an energy functional for dimeric molecules on a square lattice has been introduced and an analogy to Ising behavior has been identified [24].

The goal of the present paper is to elaborate a theoretical framework for colloidal molecular crystals in external fields built from a statistical mechanics perspective, as outlined in [26]. First, to deal with the complexity of the system we focus on the essential low-energy degrees of freedom of colloidal suspension. From the experimental observation one infers that the groups of colloids are to be considered as rather rigid entities as soon as the laser potential exceeds several tens of $k_{B} T$. The entities are referred to as molecules or dimers, trimers, etc. The key idea is to keep only discrete orientational states of the molecule as fluctuating variables, i.e., relevant for a statistical mechanics model. The separation of energy scales, e.g., the condensation or binding energy to build such molecules is much larger than the thermal scale, suggests to ignore processes connected with the breaking apart of the structure. The theoretical description we wish to develop is of phenomenological nature, since it does not explain the formation of molecules. In particular, the regime of low laser intensities where the molecular crystal exhibits a significant fraction of defects is not within the scope of our approach. The benefit of focusing on effective low-energy excitations is that one can derive simple models that are amenable to powerful methods of the statistical mechanics toolbox. In particular, it allows us to identify the appropriate broken symmetry phases and to map the problem to related magnetic transitions for which a great wealth of knowledge has been developed.

Specifically, in Sec. II we develop a theory for the experiment performed by Brunner and Bechinger [22]. We identify the relevant excitations of the system at the thermal scale as single trimer flips. A Hamiltonian is constructed that couples nearest neighboring trimers via their respective orientations. The microscopic interactions, that are, for example, also responsible for the formation of the molecules, enter the theory only implicitly through three energy scales. The explicit link to the experimental parameters, e.g., salt concentration, ef- 
(a)

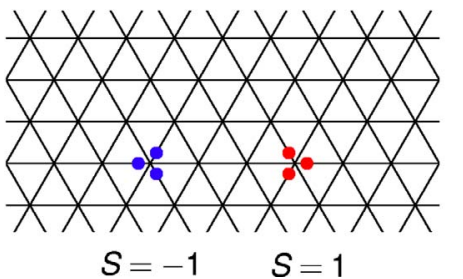

FIG. 2. (Color online) The model system. (a) The triangular lattice of the external field and the two discrete orientational states of the trimers. (b) Three interaction energies for the orientational configurations of neighboring trimers.

fective surface charge, etc., is deferred to the Appendix. It turns out that the statistical mechanics of trimers on a triangular lattice maps to an Ising model once the spectrum of single trimer excitations are evaluated. A comparison to experimental results corroborates our approach.

In Sec. III we propose an experimental setup using a triangular lattice with a filling factor 2 . Then dimers are the composite objects and three orientational states per lattice site emerge. The corresponding Hamiltonian can be understood as a natural extension of a three-states Potts model with the peculiarity that orientational pair interactions depend on the lattice orientation. We find a rich scenario of four broken symmetry phases occupying different regions in the phase diagram. In addition to "ferro-" and "antiferromagnetic" structures a herringbone structure appears and an exotic phase referred to as Japanese 6 in 1 (J6/1) is discovered. An analytical approach based on a variational mean-field calculation is presented to explore the phase boundaries. Extensive Monte Carlo simulations are employed to corroborate our findings and we present a careful discussion of the different phases.

In the Conclusion, we summarize our main results and put them in a broader perspective of experimental and theoretical solid state physics.

\section{TRIMERS}

For densities of the colloidal particles adjusted such that there are three particles per potential minimum, trimers will form spontaneously at sufficiently strong laser intensity. Then the low-energy effective degrees of freedom are given by the discrete orientational states of the composite object. Due to the symmetry of the optical lattice the trimers constitute equilateral triangles which are aligned with the triangular lattice. From Fig. 2 one infers two energetically equivalent orientational states of the trimer corresponding to the optimal balance between the compression of the constituent colloids due to the laser potential and their mutual screened Coulomb repulsion. The residual interaction between trimers depends on their relative orientation as well as on their relative lattice position. Since the residual interaction is solely due to a screened Coulomb interaction the magnitude of this energy decreases rapidly with increasing distance. For typical experimental setups the screening length is an order of magnitude smaller than the lattice constant [22], which allows us to restrict the residual interaction to nearest neigh-

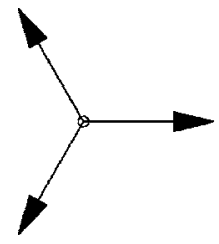

$\alpha=1$

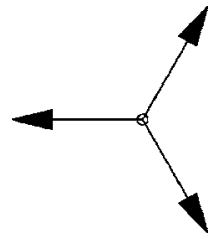

$\alpha=-1$
FIG. 3. The two classes of equivalent bond vectors in the trimer system. Note that the classes have the same symmetry as the corresponding trimer state, i.e., for $S_{i}=\alpha$.

bors. For each neighboring pair of trimers there are four possible geometrical configurations, two of which are mirror images of each other, see Fig. 2. These configurations introduce three energy scales, with generic ordering $E_{1}<E_{2}$ $<E_{3}$. These scales are functions of the screening length, the strength of the laser potential, as well as the effective charges of the constituent colloids. The Appendix provides a derivation of the explicit relation and supplements a careful discussion of the concept of composite objects.

The considerations of the previous paragraphs allow us to define the model in terms of a statistical mechanics problem. Each trimer $i$ is attributed a "spin state" $S_{i}= \pm 1$, the total number of orientational configurations being given by $2^{N}$ for a triangular lattice of $N$ sites. The phase behavior of the trimer system is encoded in a "spin Hamiltonian," which reduces to a sum over local interaction energies. The trimer system exhibits a peculiarity in the sense that these energies are dependent on the bond vector, i.e., the direction of the connecting lattice vector, in addition to the two spin orientations of the neighboring spins. Explicitly, a simultaneous flip of both spin is not a symmetry operation of the Hamiltonian, however, an additional rotation of the corresponding bond vector by $60^{\circ}$ restores the original configuration. Consequently, a rotation of the bond vector by $120^{\circ}$ with identical spin states results in the same interaction energy. Therefore, similar to the two orientational states of a trimer, there are only two inequivalent bond vectors, see Fig. 3. The Hamiltonian can be expressed in the following form:

$$
\mathcal{H}=\sum_{\alpha} \sum_{\langle i j\rangle_{\alpha}} h\left(S_{i}, S_{j} ; \alpha\right)
$$

where $\alpha= \pm 1$ denotes the orientation of the bond vector, and the symbol $\langle i j\rangle_{\alpha}$ indicates the neighboring pair of spins with directed bond vector $\alpha$. Hence each bond appears precisely once in the entire sum. Reversing the order of the lattice sites implies an inversion of the bond vector $\langle i j\rangle_{\alpha}=\langle j i\rangle_{-\alpha}$. Explicitly, the local energy functionals read

\begin{tabular}{|c|cc|}
$h\left(S_{i}, S_{j} ; 1\right)$ & \multicolumn{2}{c}{$h\left(S_{i}, S_{j} ;-1\right)$} \\
\hline$S_{i} \backslash^{S_{j}}$ & 1 & -1 \\
\hline 1 & $E_{2}$ & $E_{3}$ \\
-1 & $E_{1}$ & $E_{2}$ \\
\hline
\end{tabular}$\quad$\begin{tabular}{rr|rr|}
$S_{i} \backslash^{S_{j}}$ & 1 & -1 \\
\hline 1 & $E_{2}$ & $E_{1}$ \\
-1 & $E_{3}$ & $E_{2}$ \\
\hline
\end{tabular}


(a)

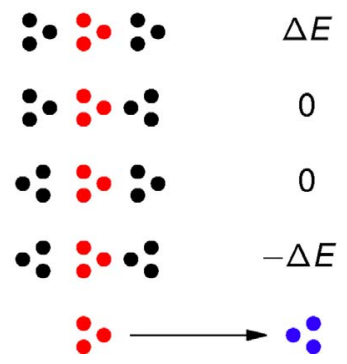

(b)

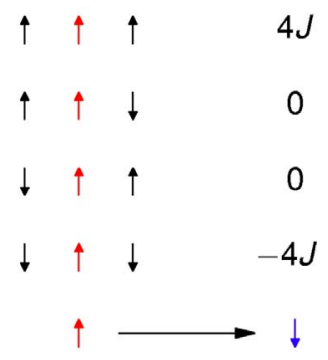

FIG. 4. (Color online) (a) Schematic representation of all possible situations for a trimer flip with the corresponding energy changes; $\Delta E=E_{1}+E_{3}-2 E_{2}$. (b) The same as in (a) but for spins of the spin-1/2 Ising Hamiltonian $\mathcal{H}=-J \Sigma_{\langle i j\rangle} S_{i} S_{j}$.

Note that due to the above explained dependence on the orientation of the bond vector, the corresponding energy "matrices" are not symmetric.

A global shift of the energy scale does not affect the phase diagram, leaving two independent energy differences. Introducing the thermal scale $k_{B} T$ allows us to construct two dimensionless parameters, and naïvely one would expect a two-dimensional phase diagram. However, we shall show now that due to geometrical constraints there is a one-to-one correspondence of the excitation spectrum of the colloidal trimers and an Ising model on a triangular lattice. Since the interaction energy is additive, it is sufficient to consider the case of three collinear trimers-i.e., considering the smallest neighborhood of trimers with representatives of both inequivalent bond orientations. If we fix the central trimer there are $2^{2}$ possible configurations of the two neighbors, all of them are depicted in Fig. 4(a). Then, flipping the central trimer yields the energy changes $\pm \Delta E, 0$ where we have defined $\Delta E \equiv E_{1}+E_{3}-2 E_{2}$. Although unexpected due to the bond-oriented local interaction energies, the trimer system reduces to an Ising model, i.e., with spins allowing for two distinct orientations and a single energy scale $\Delta E$ for excitations, see Fig. 4(b). As a result of these considerations, the Hamiltonian of Eq. (1) is equivalent to

$$
\mathcal{H}=-J \sum_{\langle i j\rangle} S_{i} S_{j}+\mathcal{H}_{0},
$$

where $\Sigma_{\langle i j\rangle}$ indicates the sum over all pairs of neighboring spins, irrespective of the orientation of the pair with respect to the lattice. The exchange energy $J$ sets the scale for local excitations and is related to the three interaction energies by

$$
J=\frac{1}{4} \Delta E=\frac{1}{4}\left(E_{1}-2 E_{2}+E_{3}\right) .
$$

The additive constant can be evaluated to $\mathcal{H}_{0}=-\frac{3}{4} N\left(E_{1}\right.$ $\left.+2 E_{2}+E_{3}\right)$. Although the energy scales possess the generic ordering $E_{1}<E_{2}<E_{3}$, the sign of the exchange coupling $J$ can attain in principle both negative and positive values. For two-dimensional colloidal systems interacting via strongly screened Coulomb interaction explicit evaluation yields positive values, i.e., "ferromagnetic" coupling. For systems with different microscopic interactions, e.g., with paramagnetic

particles, an "antiferromagnetic" phase could possibly be realized.

Let us emphasize that the mapping of the trimer Hamiltonian to an Ising model is different from the 1:1 correspondence of a lattice gas model for a binary alloy to an Ising system. There the nearest-neighbor interaction energy is parametrized also by three energy scales $\epsilon_{A A}, \epsilon_{A B}, \epsilon_{B B}$ corresponding to a pair of two neighboring $A$ atoms, a mixed pair of an $A$ and a $B$ atom, and two $B$ atoms. For the mixed pair it is irrelevant whether atom $A$ or $B$ is left and right or up and down. For the trimer problem the configurations where trimers face each other or are back-to-back are energetically rather different, see Fig. 2(b).

For a two-dimensional Ising model an exact solution is available for various lattice symmetries [27]. On a triangular lattice the transition between the "ferromagnetic" and "paramagnetic" phase occurs at $k_{B} T_{c}=4 J / \ln 3$. In the experiments of Ref. [22], the temperature is kept fixed and the intensity of the external potential is varied. This variation is accompanied by the change of the linear extensions of trimers, see the Appendix, which further affects the strength of the exchange energy $J$. Above some critical laser intensity the exchange energy becomes smaller than the critical one,

$$
J<J_{c}=k_{B} T \frac{\ln 3}{4}
$$

and the orientational order of the trimer system is lost.

The mechanism of the order-disorder transition may be paraphrased as follows: The increase of the laser potential does not affect the colloid-colloid interaction, however, it affects the effective trimer-trimer interaction. The size of the composite objects is namely determined by the balance between the colloid-colloid screened Coulomb interaction and the external laser potential. An increase of the laser potential yields a stronger confinement of the colloids to the corresponding laser spot (lattice site) and thus decreases the size of the composite objects. Since the interaction of neighboring composites depends exponentially on the distance of their respective constituents, the effective interaction between trimers weakens as the laser field is increased. At sufficiently large intensity a thermodynamic phase transition to an orientationally disordered phase occurs.

Employing experimental parameters from Ref. [22], 1/ $\kappa$ $=570 \mathrm{~nm}$ for the inverse screening length, $E_{0}=3.4 \times 10^{4} k_{B} T$ for the strength of the screened Coulomb interaction, and $T$ $=295 \mathrm{~K}$ as room temperature (see also the Appendix) we have determined the phase diagram in the $\left(V_{0} / k_{B} T,(\kappa a)^{-1}\right)$ plane and compared it to the experimental results. Brunner and Bechinger report that for $V_{0}=60 k_{B} T$ the trimer system (realized for a lattice constant $a=11.5 \mu \mathrm{m}$ ) is orientationally ordered, whereas it is disordered for $V_{0}=110 k_{B} T$. The critical strength of the laser potential found in our calculations is in striking agreement with these observations, i.e., $V_{0 c}$ $=78.6 k_{B} T$, see also Fig. 5. This agreement may appear a little fortunate due to various experimental uncertainties. Furthermore, since the sample is confined by a boundary, one would expect a rapid crossover rather than a sharp transition. The presence of grain boundaries in some experimental systems 


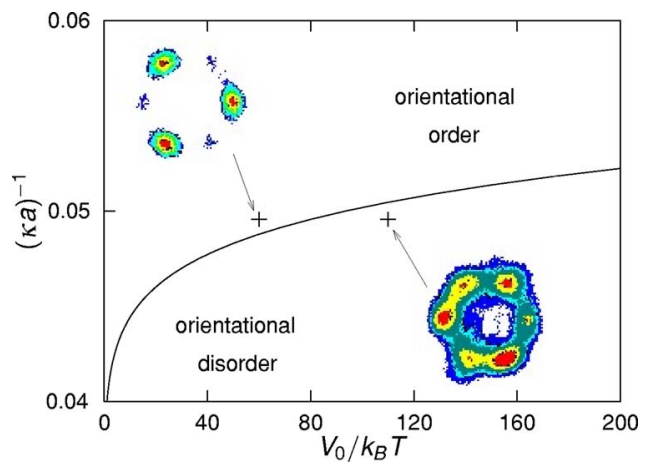

FIG. 5. (Color online) Phase diagram of the colloidal trimer system as a function of the strength of the external potential (in units of $k_{B} T$ where $T=295 \mathrm{~K}$ ) and colloid-colloid interaction characterized by a product of the inverse Debye length and the lattice constant (solid line). The two crosses correspond to the experimental observations reported in Ref. [22]. Insets: averaged local particle densities as represented in Figs. 2(f) and 2(h) of Ref. [22].

has been interpreted as a signature of a first order transition. It is reasonable to assume that the critical fluctuations are observable only in a small region of the parameter space. A more systematic study, similar to what has been performed in the case of $1 \mathrm{D}$ periodic laser potentials [28], would be highly desirable, in particular, in the regime of larger lattice constants and smaller screening lengths where the shape of the phase boundary is more sensitive to control parameters.

We have also compared our theory to Langevin dynamics simulation results of Reichhardt and Olson [23]. The phase diagram is exhibited in Fig. 6 in the $\left(V_{0} / \mathcal{E}_{0}, T / T_{m}^{0}\right)$ plane. Although the simulation has been performed for dimers on a square lattice, the basic mechanism of the orientational melting - and thus the corresponding behavior of the phase boundaries - is robust with respect to the symmetry of the composite objects and the underlying lattice. Note that the parameter $V_{0} / \mathcal{E}_{0}$ is by two orders of magnitude smaller than in the corresponding experimental setup; nevertheless, our analytical solution captures the main features of the phase boundary, i.e., shape and parameter range. The remaining phase boundaries presented in the paper of Reichhardt and

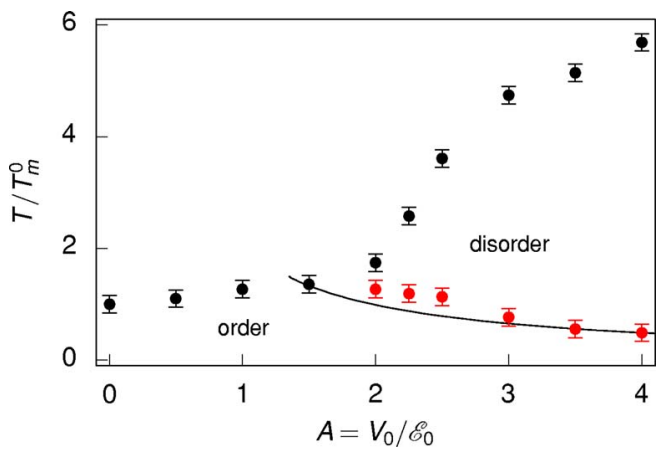

FIG. 6. (Color online) Phase diagram of the colloidal trimer system as a function of the reduced strength of the external potential and temperature. Our model describes part of the phase diagram corresponding to the orientational melting-solid line. Symbols correspond to the results from the simulation of Reichhardt and Olson, see Ref. [23], Fig. 4.

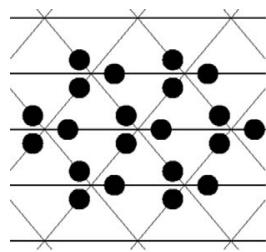

$50^{\circ}$

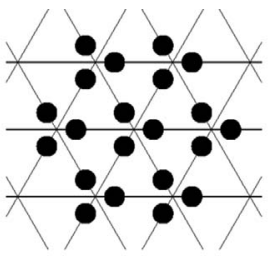

$60^{\circ}$

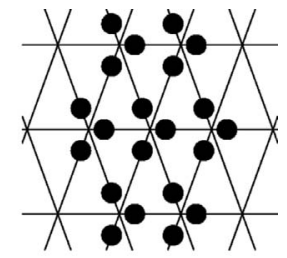

$70^{\circ}$
FIG. 7. Schematic representation of the lattice with one trimer and his neighbors for isotropic triangular lattice (middle) and uniaxially deformed ones; below the angle between the horizontal bond and the nonhorizontal one. Due to the symmetry, the exchange coupling in the horizontal direction differs from the one in the remaining directions.

Olson are connected to the fission of the composite objects and are beyond the scope of our model.

Let us comment on the possibility to impose a small uniaxial strain on the triangular lattice by varying the angles of the incident laser beams. For small deformations the trimers will merely stretch leaving the system still with two orientational states per lattice site, see Fig. 7. Since the distances between pairs of trimers are now different in different directions, the pair interactions become direction dependent. The analysis for the excitations energies is now valid separately for each direction of the three collinear trimers. For the direction perpendicular to the uniaxial deformation the exchange energy differs from the remaining two, $J_{a}$ and $J_{b}$, respectively. The system is still represented by a twodimensional Ising Hamiltonian, however, with anisotropic exchange energies. This system again allows for an exact solution, in particular, the critical temperature is now determined by the equation [27]

$$
e^{-4 J_{a} / k_{B} T}+2 e^{-4 J_{b} / k_{B} T}=1 .
$$

In conclusion, a small uniaxial strain leads only to a small shift of the critical temperature but does not lead to any qualitatively new phase behavior.

\section{DIMERS}

In this section we generalize the concepts introduced for trimers and discuss the possibility of dimeric molecular crystals and their corresponding phase diagram. Inclining the incident laser beams with respect to the plane of the twodimensional colloidal system one can adjust the lattice constant of the laser potential. Then, by appropriate matching of the lattice constant to the colloidal particle density, one can achieve an average of two particles per potential minimum. For sufficiently large laser intensity, one expects that a defect-free structure is formed, i.e., the composite objects of the system are dimers. The symmetry of the lattice allows now for three different orientational states per lattice site denoted by $\sigma_{i}=1,2,3$, see Fig. 8 (a).

\section{A. Dimer Hamiltonian}

As in the case of trimers, the interaction of neighboring dimers is the origin of orientational ordering, whereas the balance of the internal repulsion and the laser pressure 
(a)

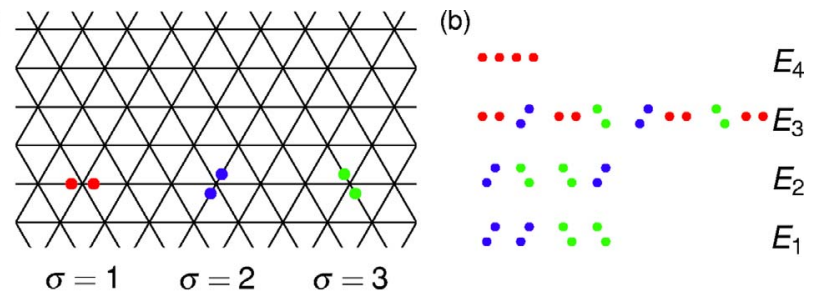

(c)

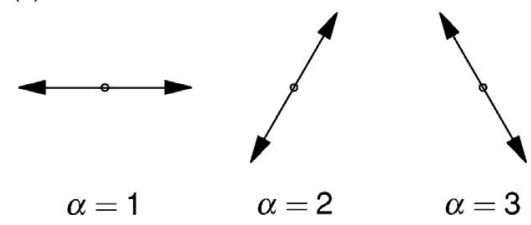

FIG. 8. (Color online) The model system. (a) The triangular lattice of the external field and the three discrete orientational states of the dimers. (b) Four interaction energies for the orientational configurations of neighboring dimers. (c) The three classes of equivalent bond vectors in the dimer system. The groups have the same symmetry as the corresponding dimer state, i.e., for $\sigma_{i}=\alpha$.

merely yields the binding energy of the dimers. From Fig. 8(b) one infers that there are four interaction energies for the $3^{2}$ configurations of neighboring dimers with generic ordering $E_{1}<E_{2}<E_{3}<E_{4}$. Explicit formulas for the relation to experimental parameters are presented in the Appendix. The dependence of the interaction of dimers on the orientation of the bond vector follows from the symmetry of the composite objects, i.e., there are three inequivalent pairs of lattice orientations $\alpha=1,2,3$, depicted in Fig. 8(c). Since each pair of equivalent lattice orientations is now collinear, reversing the order of sites in a directed bond $\langle i j\rangle_{\alpha}$ yields a directed bond within the same equivalence class. This implies that the local interaction energy is symmetric with respect to exchange of the dimers.

The total Hamiltonian of the two-dimensional dimer system corresponds to a sum over all interactions of neighboring dimer states. Denoting by $\langle i j\rangle_{\alpha}$ the pair of nearest neighbors $i, j$ whose bond vector is parallel to the orientation of the dimer state $\alpha$, the colloidal dimer Hamiltonian can be expressed in the following form:

$$
\mathcal{H}=\sum_{\alpha=1}^{3} \sum_{\langle i j\rangle_{\alpha}} h\left(\sigma_{i}, \sigma_{j} ; \alpha\right) .
$$

The direction-dependent local energy functionals $h\left(\sigma_{i}, \sigma_{j} ; \alpha\right)$ are given by

$h\left(\sigma_{i}, \sigma_{j} ; 1\right)$
\begin{tabular}{|l|lll|l|lll|l|lll|}
\hline$\sigma_{i}{ }^{\sigma_{j}}$ & 1 & 2 & 3 & $\left.\sigma_{i}\right)^{\sigma_{\jmath}}$ & 1 & 2 & 3 & $\left.\sigma_{i}\right)^{\sigma_{\jmath}}$ & 1 & 2 & 3 \\
\hline 1 & $E_{4}$ & $E_{3}$ & $E_{3}$ & 1 & $E_{1}$ & $E_{3}$ & $E_{2}$ & 1 & $E_{1}$ & $E_{2}$ & $E_{3}$ \\
2 & $E_{3}$ & $E_{1}$ & $E_{2}$ & 2 & $E_{3}$ & $E_{4}$ & $E_{3}$ & 2 & $E_{2}$ & $E_{1}$ & $E_{3}$ \\
3 & $E_{3}$ & $E_{2}$ & $E_{1}$ & 3 & $E_{2}$ & $E_{3}$ & $E_{1}$ & 3 & $E_{3}$ & $E_{3}$ & $E_{4}$ \\
\hline
\end{tabular}

Note that the $h\left(\sigma_{i}, \sigma_{j} ; \alpha\right)$ are symmetric with respect to the diagonal, and that they are related to each other through cyclic permutations, i.e., from $\alpha=1$ to $\alpha=2$ and from $\alpha=2$ to $\alpha=3$ dimer states have to be permuted cyclically, $1 \rightarrow 2$ $\rightarrow 3 \rightarrow 1$.

As noted in [26], the four interaction energies do not enter the phase diagram independently, but only two linear combinations of these are relevant for the spectrum of excitations. Introducing the thermal scale $k_{B} T$, a two-dimensional phase diagram characterizes the complete orientational ordering scenario of the dimer system. Here we provide the full chain of arguments leading to this reduction of control parameters. First it is convenient to sum over the three inequivalent lattice orientations separately, i.e., to split the Hamiltonian into $\mathcal{H}=\Sigma_{\alpha} \mathcal{H}_{\alpha}$ with $\mathcal{H}_{\alpha}=\sum_{\langle i j\rangle_{\alpha}} h\left(\sigma_{i}, \sigma_{j} ; \alpha\right)$. Then the corresponding Hamiltonian for each direction can be rewritten in a "spin nomenclature" as a generalized spin-1-Ising or generalized Potts model. Here we present the derivation for $\alpha=1$, the results for the remaining directions follow by cyclic permutations. The local energy functional can be represented as a sum of four simpler functionals,

$$
h\left(\sigma_{i}, \sigma_{j} ; 1\right)=\sum_{r=1}^{4} h_{r}\left(\sigma_{i}, \sigma_{j} ; 1\right),
$$

where the $h_{r}\left(\sigma_{i}, \sigma_{j} ; 1\right) \equiv h_{r}$ are given in terms of

$$
h_{1}: \begin{array}{|l|ccc|}
\hline \sigma_{i} \backslash^{\sigma_{j}} & 1 & 2 & 3 \\
\hline 1 & -K & 0 & 0 \\
2 & 0 & -K & 0 \\
3 & 0 & 0 & -K \\
\hline
\end{array} h_{2}: \begin{array}{|l|lll|}
\sigma_{i} \backslash^{\sigma_{j}} & 1 & 2 & 3 \\
\hline 1 & 2 L & L & L \\
2 & L & 0 & 0 \\
3 & L & 0 & 0 \\
\hline
\end{array}
$$

$h_{3}:$\begin{tabular}{|l|ccc}
\hline$\left.\sigma_{i}\right\rceil^{\sigma_{j}}$ & 1 & 2 & 3 \\
\hline 1 & $-M$ & 0 & 0 \\
2 & 0 & 0 & 0 \\
3 & 0 & 0 & 0 \\
\hline
\end{tabular}$\quad h_{4}:$\begin{tabular}{|lllll}
$\sigma_{i} l^{\sigma_{j}}$ & 1 & 2 & 3 \\
\hline 1 & $E_{2}$ & $E_{2}$ & $E_{2}$ \\
2 & $E_{2}$ & $E_{2}$ & $E_{2}$ \\
3 & $E_{2}$ & $E_{2}$ & $E_{2}$ \\
\hline
\end{tabular}

and each contribution is parametrized in terms of a single energy scale,

$$
\begin{gathered}
K=-\left(E_{1}-E_{2}\right), \\
L=E_{3}-E_{2}, \\
M=-\left(E_{4}-E_{1}-2 E_{3}+2 E_{2}\right) .
\end{gathered}
$$

Due to the generic ordering of the interaction energies of colloidal dimers, the parameters $K$ and $L$ are expected to be positive, while $M$ may attain both signs in general. The functionals allow for a direct interpretation: First, $h_{1}$ favors dimers to be in the same state, second, $h_{2}$ gives an extra 
contribution if a dimer is aligned with the bond vector connecting the dimers. Third, $h_{3}$ accounts for the high symmetry configuration, viz. both dimers aligned with the bond vector. Fourth, $h_{4}$ is independent of the configuration and corresponds to global total shift of the energy. The functionals $h_{r}\left(\sigma_{i}, \sigma_{j} ; \alpha\right)$ are easily reexpressed in terms of Kronecker symbols, and one obtains for the local energy functional

$$
h\left(\sigma_{i}, \sigma_{j} ; \alpha\right)=-K \delta_{\sigma_{i}, \sigma_{j}}+L\left(\delta_{\sigma_{i}, \alpha}+\delta_{\sigma_{j}, \alpha}\right)-M \delta_{\sigma_{i}, \alpha} \delta_{\sigma_{j}, \alpha}+E_{2} .
$$

Collecting results the total Hamiltonian reads

$$
\begin{aligned}
\mathcal{H}= & \sum_{\alpha=1}^{3} \sum_{\langle i j\rangle_{\alpha}}\left[-K \delta_{\sigma_{i}, \sigma_{j}}-M \delta_{\sigma_{i}, \alpha} \delta_{\sigma_{j}, \alpha}\right]+\sum_{\alpha=1}^{3} \sum_{\langle i j\rangle_{\alpha}}\left[L \left(\delta_{\sigma_{i}, \alpha}\right.\right. \\
& \left.\left.+\delta_{\sigma_{j}, \alpha}\right)+E_{2}\right] .
\end{aligned}
$$

The second sum evaluates to a constant once the sum over all pairs and directions is performed, $\Sigma_{\alpha} \Sigma_{\langle i j\rangle_{\alpha}}\left[L\left(\delta_{\sigma_{i}, \alpha}+\delta_{\sigma_{i}, \alpha}\right)\right.$ $\left.+E_{2}\right]=N\left(E_{2}+2 E_{3}\right)$. The phase behavior is unaffected by this global shift of energy, thus we discard the constant, and after rearranging terms, one arrives at the final expression for colloidal dimer Hamiltonian

$$
\mathcal{H}=-K \sum_{\langle i j\rangle} \delta_{\sigma_{i}, \sigma_{j}}-M \sum_{\alpha=1}^{3} \sum_{\langle i j\rangle_{\alpha}} \delta_{\sigma_{i}, \alpha} \delta_{\sigma_{j}, \alpha} .
$$

The excitation spectrum of orientational flips is governed solely by the two energy scales $K, M$ to be compared to the thermal one $k_{B} T$, and the phase diagram reduces to a twodimensional plane. The $K$ term accounts for the energy gained if two neighboring dimers are in the same orientational state, see Fig. 8(b), $E_{4}$ or $E_{1}$. The $M$ term distinguishes the high symmetry state of alignment of the dimers with the lattice bond vector, Fig. 8(b), $E_{4}$. This coupling between spin configuration and orientation of the bond vector has a close analog in the magnetic dipole-dipole interaction of localized spins on a lattice.

The spin Hamiltonian of Eq. (14) can be interpreted as a generalization of a $q$-state Potts model, the natural extension of the Ising model. In these systems, each "spin" $i$ can be in one of the $\sigma_{i}=1, \ldots, q$ equivalent states. The only energetic contribution occurs if two neighboring spins are in the same state, i.e.,

$$
\mathcal{H}=-J \sum_{\langle i j\rangle} \delta_{\sigma_{i}, \sigma_{j}}
$$

It is obvious that the colloidal dimer Hamiltonian reduces to a three-state Potts model if $M=0$.

There have been intensive studies of the critical properties of ferromagnetic, $J>0$, as well as antiferromagnetic, $J<0$, Potts models for various lattice geometries in two and three dimensions. In particular, a rigorous solution of the ferromagnetic Potts model in two dimensions is available $[29,30]$ for a square, triangular, and honeycomb lattice for $q=2$ (Ising) and $q \geqslant 4$. The melting of the ferromagnetic phase to the paramagnetic one occurs via a continuous phase transition for the case of $q=2,4$ and via a discontinuous one for $q>4$. For both cases the exact value of the critical tempera- ture for the triangular lattice is given by $[27,30]$

$$
\begin{aligned}
& \exp \frac{J}{k_{B} T_{c}}=2 \cos \left[\frac{2}{3} \cos ^{-1}\left(\frac{\sqrt{q}}{2}\right)\right] ; \quad q=2,4, \\
& \exp \frac{J}{k_{B} T_{c}}=2 \cosh \left[\frac{2}{3} \cosh ^{-1}\left(\frac{\sqrt{q}}{2}\right)\right] ; \quad q \geqslant 4 .
\end{aligned}
$$

Unfortunately, for the interesting case of $q=3$ neither the method of transfer matrices (for the Ising model) nor the circle theorem [27] for the vertex model (for $q \geqslant 4$ Potts models) is applicable. Nevertheless, since the critical point in Eq. (16) agrees with the exact Ising result, it is expected that it holds also for $q=3$. There is also strong numerical evidence by Monte Carlo simulations [30,31] that the critical temperature is still given by Eq. (16). Furthermore, Monte Carlo simulations as well as renormalization group analysis corroborate a continuous transition scenario [30] for $q=3$.

It is interesting to note that a standard mean-field analysis gives a qualitatively wrong result as far as the nature of the transition is concerned, i.e., a discontinuous scenario is predicted for $q \geqslant 3$. The value of the critical temperature is overestimated,

$$
k_{B} T_{\mathrm{MF}}=\frac{3 J}{2 \ln 2},
$$

with respect to the tentatively exact value $k_{B} T_{c}$ $=J / \ln [2 \cos (\pi / 9)]$ by a factor of 1.3654 similar to the case of the two-dimensional (2D) Ising model. Since we recover the three-state Potts model as a special case of the colloidal dimer Hamiltonian, Eq. (14), one expects that the mean-field analysis fails to predict the correct nature of the transitions also in the case of the colloidal dimer Hamiltonian.

For negative exchange coupling $J$ with a low-temperature antiferromagnetic state there are no exact solutions known. For triangular lattices renormalization group (RG) studies [32], series analysis [33], and Monte Carlo simulations [34] give for the critical point a numerical estimate $k_{B} T_{c}$ $\approx J / \ln (0.206)$. On the other hand, there is no general consensus on the nature of the transition. Extrapolated series expansions and Monte Carlo simulations find the transition to be discontinuous, whereas renormalization group analysis yields a continuous phase change [30].

\section{B. Mean-field description}

A rigorous solution of the colloidal dimer problem is certainly desirable, however, it would imply an analytic solution of the three-state Potts model as a special case, a problem that remains unsolved despite considerable efforts [27,30]. Approximate methods are required to gain further insight into the phase behavior. Mean-field theory has proven a powerful tool that allows us to identify the global topology of the phase diagram, in particular, the coexistence of phases of different broken symmetries, as well as to provide approximate numbers for the location of phase boundaries. The results of such a mean-field analysis also provide a useful reference for a numerical simulation by Monte Carlo methods which will be presented in Sec. III C. 
We use the framework of variational mean-field theory and determine the optimal distribution of dimer states within a restricted class of density matrices [1]. The normalized equilibrium density matrix $\rho=Z^{-1} \exp (-\beta \mathcal{H})$, with inverse temperature $\beta=1 / k_{B} T$, is difficult to obtain since the partition sum $Z=\operatorname{Tr}[\exp (-\beta \mathcal{H})]$ usually cannot be evaluated exactly. To overcome this problem it is favorable to approximate $\rho$ by a product of single-site density matrices $\rho_{i}$,

$$
\rho\left(\left\{\sigma_{i}\right\}\right)=\prod_{i} \rho_{i}\left(\sigma_{i}\right),
$$

i.e., spins at different sites are taken as uncorrelated. The quantities $\rho_{i}\left(\sigma_{i}\right)$ represent the probability to find the dimer at site $i$ in state $\sigma_{i}$. Normalization implies in particular $\Sigma_{\sigma_{i}} \rho_{i}\left(\sigma_{i}\right)=1$. The free energy corresponding to a system represented by a density matrix as in Eq. (18) results from the usual balance energy vs entropy $\mathcal{F}_{\rho}=\mathcal{E}_{\rho}-T \mathcal{S}_{\rho}$, where the mean energy $\mathcal{E}_{\rho}$ and the entropy $\mathcal{S}_{\rho}$ are evaluated by the rules of statistical mechanics,

$$
\mathcal{E}_{\rho}=\langle\mathcal{H}\rangle_{\rho}, \quad \mathcal{S}_{\rho}=-k_{B}\langle\ln \rho\rangle_{\rho},
$$

where $\langle\cdots\rangle_{\rho}$ denotes averaging with respect to the approximate distribution of Eq. (18). One can show in general that Bogolyubov's inequality [1] sets a lower bound to $\mathcal{F}_{\rho}$,

$$
\mathcal{F}_{\rho}=\operatorname{Tr}[\rho \mathcal{H}]+k_{B} T \operatorname{Tr}[\rho \ln \rho] \geqslant \mathcal{F} .
$$

Here $\mathcal{F}$ denotes the exact thermodynamic free energy. The idea of the variational mean-field method is to find the single-particle $\rho_{i}\left(\sigma_{i}\right)$ that yields the variational mean-field free energy $\mathcal{F}_{\rho}$ closest to the thermodynamic one, viz. $\mathcal{F}$.

For the colloidal dimer Hamiltonian the variational meanfield free energy reads

$$
\begin{aligned}
\mathcal{F}_{\rho}= & -K \sum_{\langle i j\rangle} \sum_{\sigma} \rho_{i}(\sigma) \rho_{j}(\sigma)-M \sum_{\alpha} \sum_{\langle i j\rangle_{\alpha}} \rho_{i}(\alpha) \rho_{j}(\alpha) \\
& +k_{B} T \sum_{i} \sum_{\sigma} \rho_{i}(\sigma) \ln \left[\rho_{i}(\sigma)\right] .
\end{aligned}
$$

Note that in the $M$ term the spin orientation and the lattice bond are aligned, $\alpha=\sigma$. This expression for the free energy is still subject to the minimization procedure with respect to appropriate sets of single-site density matrices.

The symmetries of the dimer states in the broken symmetry phase have to be reflected in the Ansatz for the $\rho_{i}\left(\sigma_{i}\right)$. In general, one introduces a number of sublattices each comprising a set of equivalent lattice sites with respect to orientational order.

In the simplest case, all lattice sites are equivalent and the only phase transition allowed separates a paramagnetic phase from a ferromagnetic one. The suitable density matrix is therefore site-independent,

$$
\rho_{i}\left(\sigma_{i}\right) \equiv \rho\left(\sigma_{i}\right) \mapsto \rho_{\sigma_{i}}
$$

Upon substituting this Ansatz in the mean-field free energy, Eq. (21), yields the free energy density, i.e., free energy per lattice site, $f=\mathcal{F}_{\rho} / N$,
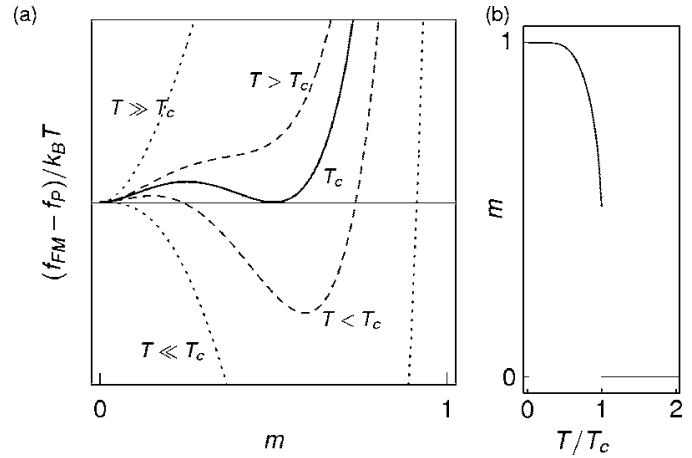

FIG. 9. (a) Free energy as a function of the magnetization for various temperatures above, below, and at the critical point. Local minima correspond to metastable phases. (b) Mean-field temperature dependence of the magnetization for the ferromagnetic phase of the colloidal dimer Hamiltonian. At the critical temperature, $T / T_{c}=1$, the order parameter exhibits a jump.

$$
f=-(3 K+M) \sum_{\sigma} \rho_{\sigma}^{2}+k_{B} T \sum_{\sigma} \rho_{\sigma} \ln \rho_{\sigma}
$$

Let us note that within the single-site mean-field description, the parameters $K$ and $M$ appear only in the combination $3 K+M$. Applying the same procedure for the three-state Potts model gives an identical expression for the free energy density provided $3 K+M$ is substituted by $J$.

A ferromagnetic (FM) phase is characterized by a broken symmetry, i.e., a particular dimer state is preferred with respect to the remaining ones. The single dimer excitations with respect to the ferromagnetic ground state are degenerate, i.e., the two possible minority orientations are equally probable. By the symmetry of the FM phase, this property is preserved in the whole phase region. Selecting the majority component as the $\sigma=1$ state, we parametrize $\rho_{1}=(1$ $+2 m) / 3$ by a generalized magnetization $m$. The minority probabilities are determined by the normalization $\Sigma_{\sigma} \rho_{\sigma}=1$ to $\rho_{2}=\rho_{3}=(1-m) / 3$. The magnetization is restricted to $-1 / 2$ $\leqslant m \leqslant 1$, and the fully aligned ferromagnetic state corresponds to $m=1$, whereas the paramagnetic one is given by $m=0$. A negative magnetization would indicate that the distinguished spin state is less probable than the remaining two. Hence there is an inherent asymmetry $m \mapsto-m$ in the meanfield description, and, as we shall see later, all transitions within this mean-field approach are (falsely) predicted to be discontinuous, similar to the case of a pure Potts model.

Substitution into Eq. (23) yields the excess free energy of the ferromagnetic phase with respect to paramagnetic one,

$$
\frac{f_{\mathrm{FM}}-f_{P}}{k_{B} T}=-\frac{2 \epsilon}{3} m^{2}+\frac{1+2 m}{3} \ln (1+2 m)+2 \frac{1-m}{3} \ln (1-m),
$$

which depends on the effective parameter $\epsilon=\beta(3 K+M)$. The corresponding graph is shown in Fig. 9(a). One infers the typical characteristics of a first order phase transition, e.g., a discontinuous change of the order parameter and metastable states. 

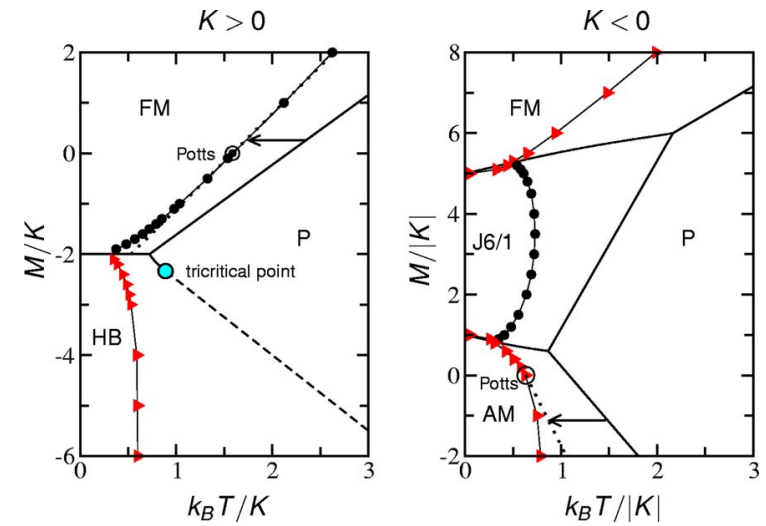

FIG. 10. (Color online) Phase diagram for colloidal dimers. Solid lines represent first order transitions derived from the mean field (MF) description and the dashed line after the tricritical point represents a line of second order transitions. Dots and triangles denote the respective continuous and discontinuous transition points as obtained by Monte Carlo (MC) simulations (lines connecting the symbols are guides to the eye); the two encircled symbols indicate the pure Potts transitions. Dotted lines represent the extrapolation of the critical point of the Potts model, see text.

The equation of state for the magnetization is obtained by minimizing $f_{\mathrm{FM}}$ with respect to $m$,

$$
2 \epsilon m=\ln \frac{1+2 m}{1-m} .
$$

One infers that $m=0$, corresponding to the paramagnetic phase, is always a solution to this equation. Depending on the value of the effective parameter $\epsilon$ nontrivial solutions may occur that exhibit lower mean-field free energies than the paramagnetic one, $f_{\mathrm{P}}=-k_{B} T(\epsilon / 3-\ln 3)$. For infinite temperatures $(\epsilon=0)$ the solution to this equation reduces to the paramagnet $(m=0)$, whereas at zero temperature $(\epsilon \rightarrow \infty)$ full alignment of the ferromagnet $(m=1)$ occurs. The complete temperature dependence of the magnetization is depicted in Fig. 9(b).

The thermodynamic transition from a paramagnetic phase $(P)$ occurs at the temperature $T_{c}$ when the nontrivial minimum of the free energy $f_{\mathrm{FM}}$ equals the one of the paramagnetic state $f_{P}$. With the help of the equation of state, Eq. (25), one finds for the critical effective parameter $\epsilon_{c}=2 \ln 2$, which implies for the critical temperature

$$
\text { FM- } P: \quad k_{B} T_{c}=\frac{3 K+M}{2 \ln 2} .
$$

Representing the phase diagram in terms of the dimensionless parameters $k_{B} T /|K|$ and $M /|K|$, the FM- $P$ phase boundary yields a straight line, see Fig. 10.

The magnetization at the critical point is finite and attains the value $m_{c}=1 / 2$. The jump in the derivative of free energy density with respect to temperature at the phase transition implies a latent heat per dimer which evaluates to $q_{l} / k_{B} T_{c}$ $=\ln (2) / 3$. The heat capacity jumps at $T_{c}$ by $\Delta c_{N}=\left(8 k_{B} / 3\right)$ $\times\left\{\ln ^{2}(2) /[3-4 \ln (2)]\right\}$. Note that these quantities are universal in the sense that they do not depend on the parameters $K$


FIG. 11. (Color online) Ground state ordered structures of 2D colloidal dimers: ferromagnetic (FM), herringbone (HB), Potts antiferromagnetic (AM), and Japanese 6 in 1 (J6/1), structure. In the latter, the energy of the system does not depend on the orientation of dimers on sites denoted by circles.

and $M$, i.e., they are constant along the whole phase boundary. The mean-field analysis appears to be qualitatively correct for $K<0$, whereas for $K>0$ the simulation results suggest that fluctuations render the phase transition to a continuous one, as will be discussed in detail in Sec. III C.

The ferromagnetic phase represents only the simplest broken symmetry. Spatially varying order parameters give rise to complex phase behavior, in particular, the sites fall into different classes of sublattices. Comparing the exact ground state energies of different ordered structures, one can infer the possible broken symmetry phase at low temperatures. In total, we have found four ordered phases of different symmetry, see Fig. 11, that are thermodynamically stable in different regions of the phase diagram, depicted in Fig. 10. In addition to the ferromagnetic one, there appears an antiferromagnetic (AM) phase stable for $K<0$ and not too large $M$. Here, in the fully ordered state, neighboring dimers are in different orientational states. On the triangular lattice this implies that each dimer is surrounded by three neighbors of a configuration rotated by $60^{\circ}$ and by three neighbors of a configuration rotated by $-60^{\circ}$ with respect to the configuration of the central dimer. There are three triangular sublattices with a lattice constant of $a \sqrt{3}$ and the sublattices are equivalent in the sense that each of them can be obtained by a spatial translation with a simultaneous rotation of the dimers of any other one. For positive $K$ and sufficiently negative $M$ a herringbone (HB) structure represents the state of lowest free energy, see Fig. 11. Statistically equivalent dimers are arranged in two sets of rows, the majority orientational states corresponding to the ones not parallel to the orientation of the rows. Finally, we have found that intervening in between the FM and AM for $K<0$ there appears a phase of four triangular sublattices. Three of them are equivalent in the sense mentioned above, the fourth one exhibiting no preferred orientation. We refer to it as Japanese 6 in 1 phase $(\mathrm{J} 6 / 1)$, due to its resemblance to a weaving pattern for chain mails worn by samurais in the 14th century [35]. 
To describe these complex phases generalized Ansätze have to be used for the density matrix. Then the site dependence of the components of $\rho_{i}\left(\sigma_{i}\right)$ is encoded in the sublattice $\Gamma$ the site $i$ belongs to, i.e.,

$$
\rho_{i}\left(\sigma_{i}\right)=\rho_{\sigma_{i}}^{\Gamma} ; \quad i \in \text { sublattice } \Gamma .
$$

Some of the density matrices $\rho_{\sigma}^{\Gamma}$ are related by permutations of dimer states. Explicitly, for the AM phase as well as for the HB the sublattices are equivalent, so that only one density matrix characterizes the order of the system. For the J6/1 phase the order in the three equivalent sublattices is determined by one nontrivial density matrix, whereas the fourth one attributes equal probabilities to all dimer orientations. Let us mention that an additional symmetry occurs for the $\mathrm{AM}$ and $\mathrm{J} 6 / 1$ phase. Here, the two minority orientations are equivalent as was the case in the FM phase. The corresponding density matrix is parametrized by a single number, a generalized magnetization $m$ defined on a sublattice. The situation is different for the HB phase. An excitation of a dimer to the majority orientation of the neighboring row is in general different from aligning the dimer with the orientation of the row. Correspondingly, the density matrix in the HB phase requires two parameters.

The three sublattices for the antiferromagnetic phase are inferred from the ground state represented in Fig. 11. The single-site density matrices corresponding to the sublattices $A, B, C$, see Eq. (27), are parametrized by

$$
\begin{gathered}
\rho_{1}^{A}=\rho_{2}^{B}=\rho_{3}^{C}=(1+2 m) / 3, \\
\rho_{2,3}^{A}=\rho_{1,3}^{B}=\rho_{1,2}^{C}=(1-m) / 3 .
\end{gathered}
$$

The excess free energy density of the antiferromagnet is then obtained by substituting this Ansatz into Eq. (21),

$$
\frac{f_{\mathrm{AM}}-f_{\mathrm{P}}}{k_{B} T}=-\frac{2 \mu}{3} m^{2}+\frac{1+2 m}{3} \ln (1+2 m)+2 \frac{1-m}{3} \ln (1-m),
$$

where the sole parameter entering this expression is given by $\mu=-\beta(3 K+M) / 2$. The shape of the free energy density $f_{\mathrm{AM}}$ as a function of temperature is identical to the one of the ferromagnetic phase $f_{\mathrm{FM}}$, see Fig. 9. The equation of state for the sublattice magnetization $m$ is thus obtained by replacing $\epsilon$ by $\mu$ in Eq. (25), i.e., $2 \mu m=\ln [(1+2 m) /(1-m)]$. The same substitution for the critical temperature, Eq. (26), yields

$$
\text { AM- } P: \quad k_{B} T_{c}=-\frac{3 K+M}{4 \ln 2} .
$$

The AM- $P$ phase boundary is represented by a straight line in the $T /|K|-M /|K|$ phase diagram, Fig. 10. The latent heat and the excess of the heat capacity are again the same as for the FM phase provided one substitutes $\epsilon$ with $\mu$.

For the Japanese 6 in 1 phase the appropriate Ansatz for the three equivalent sublattices $(A, B, C)$ is identical to the antiferromagnetic case, Eq. (28), whereas the fourth $(O)$ is degenerate,

$$
\rho_{1,2,3}^{O}=1 / 3 .
$$

The excess free energy density for the J6/1 symmetry is readily obtained,

$$
\begin{aligned}
\frac{f_{\mathrm{J} 6 / 1}-f_{\mathrm{P}}}{k_{B} T}= & \frac{3}{4}\left(-\frac{2 \nu}{3} m^{2}+\frac{1+2 m}{3} \ln (1+2 m)\right. \\
& \left.+2 \frac{1-m}{3} \ln (1-m)\right) .
\end{aligned}
$$

Here $\nu=-\beta(3 K-M) / 3$ denotes the sole effective parameter. Up to the multiplicative constant $3 / 4$ the excess free energy density of the J6/1 phase is identical to the excess free energy densities of the FM and AM phases discussed above, provided $\nu$ is substituted by $\epsilon$ or $\mu$, respectively. The prefactor $3 / 4$ arises since only three of the four sublattices exhibit a broken symmetry. The equation of state obtained as the derivative of the free energy density with respect to the sublattice magnetization $m$ is again formally identical to the cases discussed above, $2 \nu m=\ln [(1+2 m) /(1-m)]$. The critical temperature for the $\mathrm{J} 6 / 1-P$ phase transition is readily obtained via the substitution rule, yielding $\nu_{c}=2 \ln 2$ and

$$
\mathrm{J} 6 / 1-P: \quad k_{B} T_{c}=-\frac{3 K-M}{6 \ln 2} .
$$

The J6/1-P phase boundary is indicated in Fig. 10. Since only three of the four sublattices exhibit a broken symmetry, the latent heat as well as the jump of the heat capacity at the phase transition are 3/4 of the universal values of the FM- $P$ or AM- $P$ transitions.

The herringbone structure $(\mathrm{HB})$ requires two parameters, one for the majority magnetization $m$ and a second "biaxiality" parameter $n$ for the asymmetry of the minority orientations. The need for the biaxiality parameter becomes clear if one considers excitations of $\mathrm{HB}$ ground state. A single dimer flip to the orientation of the neighboring row costs an energy of $\Delta E=-2 K-2 M$, whereas aligning it with the row induces an energy change of only $\Delta E=2 K$. (Note that the single dimer excitation energies become degenerate for $M=-2 K$.) The low temperature excitations of the HB structure are represented by a dilute gas of defects of the latter kind. Considering the sign of the single-dimer excitation energies an estimate for the stability of the HB structure at low temperatures is possible, i.e., for positive $K$ and sufficiently large negative $M$.

For the parametrization of the sublattice single-site density matrices we choose

$$
\begin{gathered}
\rho_{2}^{A}=\rho_{3}^{B}=(1+2 m) / 3, \\
\rho_{1,3}^{A}=\rho_{1,2}^{B}=(1-m \pm n) / 3 .
\end{gathered}
$$

Then the free energy density corresponding to the HB symmetry reads 

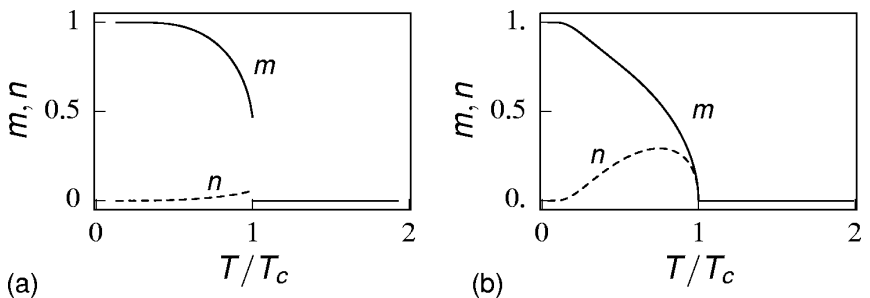

FIG. 12. Order parameters $m$ and $n$ as a function of temperature for a path of constant $M / K$. (a) A discontinuous phase transition characterized by a jump of $m$ and $n$ for $M / K=-2.1$ and (b) a continuous phase transition for $M / K=-5$.

$$
\begin{aligned}
\frac{f_{\mathrm{HB}}-f_{\mathrm{P}}}{k_{B} T}= & \frac{M}{3 k_{B} T} m^{2}-\frac{4 K+M}{9 k_{B} T} n^{2}+\frac{2(2 K+M)}{3 k_{B} T} m n \\
& +\frac{1+2 m}{3} \ln (1+2 m)+\frac{1-m+n}{3} \ln (1-m+n) \\
& +\frac{1-m-n}{3} \ln (1-m-n) .
\end{aligned}
$$

The excess free energy is subject to a simultaneous minimization with respect to both order parameters $m, n$. In general, one cannot find an analytic solution of the equations of state,

$$
\begin{gathered}
k_{B} T \ln \frac{1-m+n}{1-m-n}=-2(2 K+M) m+\frac{2}{3}(4 K+M) n, \\
k_{B} T \ln \frac{(1+2 m)^{2}}{(1-m)^{2}-n^{2}}=-2 M m-2(2 K+M) n .
\end{gathered}
$$

The generic behavior close to the HB- $P$ phase transition is illustrated in Fig. 12 for a path of constant $M /|K|$ close to the HB-FM line and one starting deep in the HB phase.

For the case of a continuous transition the transition temperature can be obtained analytically by expanding the excess free energy density in powers of the small quantities $m$ and $n$, which up to quadratic order reads

$$
\frac{f_{\mathrm{HB}}-f_{\mathrm{P}}}{k_{B} T}=\left(\frac{M}{3 k_{B} T}+1\right) m^{2}+\frac{4 K+2 M}{3 k_{B} T} m n+\left(1-\frac{4 K+M}{3 k_{B} T}\right) \frac{n^{2}}{3} \text {. }
$$

The quadratic form ceases to be positive definite and the paramagnetic phase becomes unstable via a continuous transition at the critical temperature,

$$
\text { HB- } P, \quad M \leqslant-\frac{7 K}{3}: \quad k_{B} T_{c}=-\frac{2(K+M)}{3},
$$

and the critical direction is along the diagonal $(m, n)$ $\sim(1,1)$, i.e., the order parameters become equal close to the transition, see Fig. 12. In the regime $-2 K \leqslant M \leqslant-7 K / 3$ the melting of the HB structure towards the paramagnetic phase is discontinuous. The tricritical point, where the order of the phase transition changes, can be determined again by local considerations. A suitable nonlinear transformation of variables $(m, n)$, shows that the origin becomes unstable with respect to higher order terms along the critical direction at $M=-7 K / 3, k_{B} T_{3 c}=8 K / 9$.

For $M=-2 K$ the excitation energies of the isolated singledimer flips in the HB structure become degenerate. This is reflected by the fact that the excess free energy, Eq. (35), exhibits the additional symmetry $n \rightarrow-n$ along this line. At zero temperature the HB phase is perfectly ordered $(m=1$, $n=0)$, i.e., in a $n$-symmetric phase. Increasing the temperature does not give rise to a continuous or discontinuous $n$-symmetry breaking, so $n \equiv 0$ holds along the $M=-2 K$ line for all temperatures. With this observation in mind, one checks that the mean-field free energy density of the HB phase for $M=-2 K$ equals the one of the FM phase for all temperatures. Thus the line $M=-2 K$ is a coexistence line of the two neighboring phases. The special point for the discontinuous transition to the $P$ phase, $k_{B} T_{c}=K /(2 \ln 2)$, constitutes a triple point where FM, HB, and $P$ phases coexist.

The remaining coexistence lines between different ordered phases, i.e., for $K<0$ between FM and $\mathrm{J} 6 / 1$ and between AM and J6/1, can only be determined numerically and are included in the phase diagram. Here we present in short approximate analytical solutions in terms of asymptotic lowtemperature expansions.

At the coexistence line the free energy densities of the two coexisting phases are equal. In particular, for the FM$\mathrm{J} 6 / 1$ line $f_{\mathrm{FM}}\left(m_{\mathrm{FM}}, T\right)=f_{\mathrm{J} 6 / 1}\left(m_{\mathrm{J} 6 / 1}, T\right)$, where $f_{\mathrm{FM}}$ and $f_{\mathrm{J} 6 / 1}$ are defined in Eqs. (24) and (32) and the $m$ 's are solutions of the corresponding equations of state. For $T \rightarrow 0(M /|K| \geqslant 5)$, $m_{\mathrm{FM}}, m_{\mathrm{J} 6 / 1} \approx 1$ which yields the coexistence line

$$
\mathrm{FM}-\mathrm{J} 6 / 1, M /|K| \geqslant 5: \quad k_{B} T_{c} \approx \frac{2(5 K+M)}{\ln 3} .
$$

Close to the FM-J6/1- $P$ triple point, $M=6|K|, \quad k_{B} T$ $=3|K| /(2 \ln 2)$, the magnetizations approximately take their critical values, $m_{\mathrm{FM}}, m_{\mathrm{J} 6 / 1} \sim m_{c}=1 / 2$, and give rise to

$$
\text { FM-J6/1, } \quad M /|K| \lesssim 6: \quad k_{B} T_{c}=\frac{3(5 K+M)}{2 \ln 2} .
$$

For the AM-J6/1 line an equivalent procedure with the same approximate solutions for the magnetizations close to the ground state or triple point results in

$$
\begin{gathered}
\mathrm{AM}-\mathrm{J} 6 / 1, \quad M /|K| \leqslant 1: \quad k_{B} T_{c} \approx-\frac{2(K+M)}{\ln 3}, \\
M /|K| \geqslant \frac{3}{5}: \quad k_{B} T_{c} \approx-\frac{3(K+M)}{2 \ln 2} .
\end{gathered}
$$

Analytical expressions for exact or approximate critical lines are gathered in Table I and the mean-field special lines, i.e., tricritical and triple points, in Table II.

\section{Results of the Monte Carlo simulations}

In this subsection we exemplify the phase behavior of the colloidal dimer system by numerical exact means, i.e., Monte Carlo methods. In particular, we corroborate the existence of all the phases discussed within the mean-field 
TABLE I. Phase boundaries obtained in mean-field theory. The approximate results for the J6/1-FM and J6/1-AM transition correspond to the asymptotic low-temperature (high-temperature) solution.

\begin{tabular}{ll}
\hline \hline Transition & Critical line \\
\hline$K>0$ & \\
FM- $P$ & $k_{B} T_{c}=(3 K+M) /(2 \ln 2)$ \\
HB- $P$ & $k_{B} T_{c}=-(2 / 3)(K+M), M \leqslant-7 K / 3$ \\
FM-HB & $M=-2 \mathrm{~K}$ \\
$K<0$ & \\
FM- $P$ & $k_{B} T_{c}=(3 K+M) /(2 \ln 2)$ \\
J6/1-P & $k_{B} T_{c}=(-3 K+M) /(6 \ln 2)$ \\
AM- $P$ & $k_{B} T_{c}=-(3 K+M) /(4 \ln 2)$ \\
J6/1-FM & $k_{B} T_{c} \approx 3(5 K+M) /(2 \ln 2), M \leqq 6|K|$ \\
J6/1-AM & $k_{B} T_{c} \approx 2(5 K+M) / \ln 3, M \gtrsim 5|K|$ \\
& $k_{B} T_{c} \approx-3(K+M) /(2 \ln 2), M \gtrsim 3|K| / 5$ \\
\hline \hline & $k_{B} T_{c} \approx-2(K+M) / \ln 3, M \lesssim|K|$ \\
\hline
\end{tabular}

analysis and address the nature of the phase boundaries separating the corresponding phases of broken symmetry. The results obtained within mean field thereby play a crucial role to identify the relevant order parameters to be monitored.

The standard Metropolis algorithm [36] has been employed to generate a sequence of configurations representative for the canonical ensemble to some specified temperature. The starting configuration for the lowest temperature has been chosen as the perfectly ordered (exact) ground state corresponding to the pair of energy scales $(K, M)$. The temperature has been increased gradually starting from the representative configuration of the previous parameter set. We have considered systems of different sizes subject to periodic boundary conditions. Explicitly, the number of rows $L$, each consisting of $L$ dimers, has been varied, $L=12,32,52,102$, to ensure that the results have approached the infinite system size limit. Before actual data were collected the system has been carefully equilibrated by performing up to several thousand Monte Carlo cycles. Each Monte Carlo cycle consists of $N \ln N$ [46] attempted dimer flips, where $N=L^{2}$ is the total number of dimers in the system. To ensure that the configurations entering the data analysis are statistically indepen-

TABLE II. Mean-field special points.

\begin{tabular}{ll}
\hline \hline Coexisting phases & {$\left[k_{B} T /|K|, M /|K|\right]$} \\
\hline$K>0$ & \\
FM-HB- $P$ & {$[1 /(2 \ln 2),-2]$} \\
HB- $P$ tricritical point & {$[8 / 9,-7 / 3]$} \\
$K<0$ & \\
FM-J6/1-P & {$[3 /(2 \ln 2), 6]$} \\
AM-J6/1- $P$ & {$[3 /(5 \ln 2), 3 / 5]$} \\
\hline \hline
\end{tabular}

dent, we have considered configurations separated by up to 1000 Monte Carlo cycles in the vicinity of a phase transition.

To characterize the broken symmetry phases we have considered the respective order parameters. For example, in the ferromagnetic case the fluctuating magnetization reads

$$
\mathcal{M}=\frac{\mathcal{N}_{\Sigma}}{N}-\frac{1}{2} \frac{\sum_{\sigma \neq \Sigma} \mathcal{N}_{\sigma}}{N} .
$$

Here, $\mathcal{N}_{\sigma}$ is the fluctuating number of dimers in the state $\sigma$. In particular, $\mathcal{N}_{\Sigma}$ corresponds to the majority orientation in the given configuration, and $N=\Sigma_{\sigma} \mathcal{N}_{\sigma}$ is the total number of the dimers in the system. The macroscopic magnetization $m=\langle\mathcal{M}\rangle$ is evaluated by averaging over the statistically independent configurations obtained as discussed above. We have also measured the variance, $\left\langle(\delta \mathcal{M})^{2}\right\rangle, \delta \mathcal{M}=\mathcal{M}-m$, which is related to a generalized susceptibility,

$$
\chi=\frac{1}{k_{B} T}\left\langle(\mathcal{M}-m)^{2}\right\rangle,
$$

by a fluctuation-dissipation theorem. Furthermore, we have computed the average energy $U=\langle H\rangle$ (with $u=U / N$ we will denote the average energy per dimer) as well as the corresponding variance related to the specific heat per dimer,

$$
c_{N}=\frac{1}{N k_{B} T^{2}}\left\langle(H-U)^{2}\right\rangle .
$$

For the other broken symmetries the fluctuating sublattice magnetizations are defined to reproduce the definitions given in the previous section.

In the following, the phase behavior as well as the phase transitions for the different cases will be illustrated. Since a mean-field result does not always predict the correct qualitative behavior, in particular the order of the phase transition, see Sec. III B, we have put considerable effort to investigate the nature of the phase boundaries.

\section{Ferromagnetic system}

By comparison of the free energies of different ordered configurations we have found that the ground state with the ferromagnetic broken symmetry is realized for $K>0$ with $M / K>-2$ and for $K<0$ with $M /|K|>5$. Due to the different mechanism of the ferromagnetic ordering in the two cases, the corresponding phase transitions to the paramagnetic phase differ in their nature and characteristics. In the following we will discuss the two regions separately.

(i) For $K>0$ the parallel ordering of dimers is favored by the $K$ term of the colloidal dimer Hamiltonian, Eq. (14), i.e., by its Potts part. In addition, the remaining $M$ term also favors this preference and effectively rescales the energy scale $K$ in the same way as it has resulted from the meanfield description. In essence, in this regime the colloidal dimer system can be described by an effective Hamiltonian, $\mathcal{H}_{\text {eff }}=-K_{\text {eff }} \sum_{\langle i j\rangle} \delta_{\sigma_{i}, \sigma_{j}}$, where $K_{\text {eff }}=K+M / 3$. Our Monte Carlo simulations are in agreement with the known results for such a Hamiltonian. The FM- $P$ phase transition is clearly continuous as can be inferred from the pronounced fluctuations of 
(a)

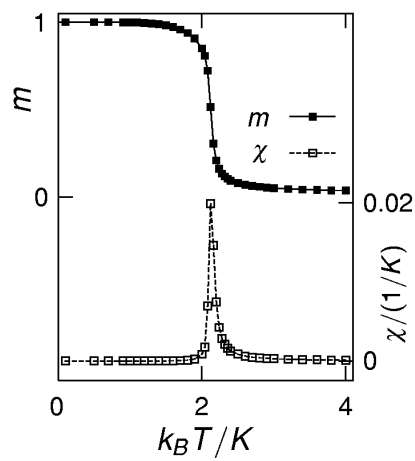

(b)

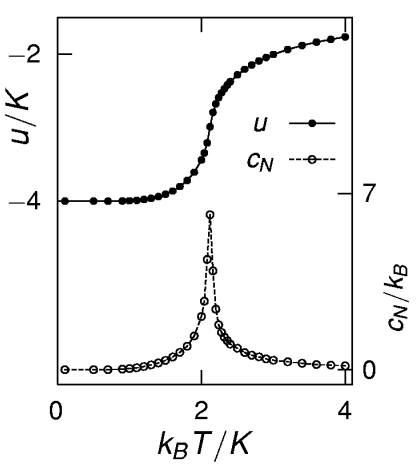

FIG. 13. The ferromagnetic system $(K>0, M / K=1)$. Temperature dependence (a) of the order parameter and its variance and (b) of the energy per dimer and its variance. Both indicate a continuous nature of the phase transition.

the magnetization and energy in the vicinity of the transition, see Fig. 13. Furthermore, the distribution functions of the magnetization and energy per dimer exhibit only a single peak in the whole temperature range under consideration (not shown).

The critical temperature of the FM- $P$ transition closely follows the "exact" value of the Potts Hamiltonian, Eq. (16), provided the exchange coupling $J$ is replaced by the above mentioned effective energy scale $K_{\text {eff }}$. Only close to the coexistence region with the $\mathrm{HB}$ phase, for $-1>M /|K|>-2$, the critical temperature deviates from the scaling law in $K_{\text {eff }}$ and the ferromagnetic order melts before the Potts critical temperature is reached, see Fig. 10. Nevertheless, within the numerical precision the nature of the transition remains unaffected.

(ii) For $K<0$ the Potts term of the colloidal dimer Hamiltonian, Eq. (14), disfavors ferromagnetic order and antiferromagnetic order would be expected. However, for high values of the energy scale $M$, explicitly for $M /|K|>5$, the term favoring the alignment of neighboring dimers along the line connecting them dominates, and the system exhibits a FM symmetry at low temperatures.

The critical properties as well as the mechanism of the transition to the $P$ phase are expected to differ from those of the Potts Hamiltonian. Indeed, the MC simulations of the full Hamiltonian reveal that the phase change is discontinuous. At the critical point the systems "jumps" between the two degenerate, FM and $P$, phases, and typical snapshots reveal phase coexistence regions at the critical point (not shown). The discontinuous nature of the transition is also evidenced by the fluctuations of the order parameter and energy, see Fig. 14.

The occurrence of metastable phases can be quantified by determining the distribution functions for the fluctuating values of the order parameter and energy per dimer [37]. We have carefully collected their distributions in the vicinity of the transition and confirm a discontinuous transition.

\section{Herringbone phase}

The broken symmetry state with a herringbone pattern is realized for $K>0$ and $M / K<-2$. Within mean-field theory

(a)

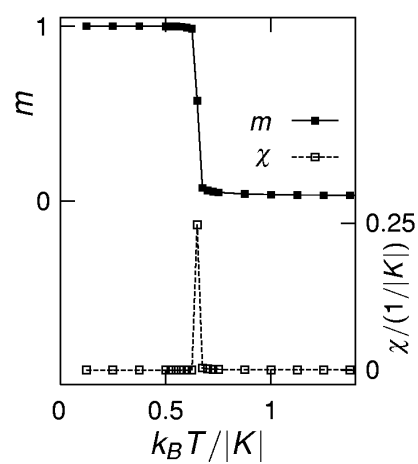

(b)

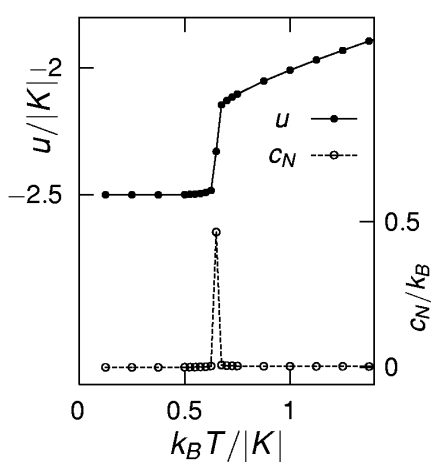

FIG. 14. The ferromagnetic system $(K<0, M /|K|=5.5)$. Temperature dependence (a) of the order parameter and its variance and (b) of the energy per dimer and its variance (right). Both indicate a discontinuous nature of the phase transition.

we have shown that its ordering is described by two order parameters, i.e., the magnetization $m$ and the biaxiality parameter $n$. In MC simulations we have monitored them both.

Qualitatively, the MC temperature dependences of $m$ and $n$ are the same as the ones determined within the mean-field description. We find a discontinuous phase change for $M / K \lesssim-2$, identified by jumps of the magnetization and energy per dimer at the critical temperature. The jump of the biaxiality parameter is masked by rounding due to the finite size of the system. However, we find a sharp increase upon approaching the critical point from below, similar to the one found within the mean-field description, see Fig. 15. For increasing negative values of $M / K$ the latent heat decreases, so that below $M / K=-4$ we cannot distinguish between a (a)

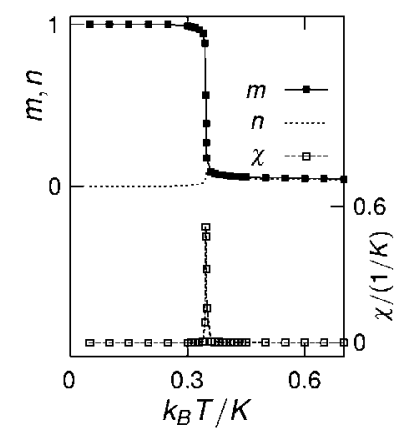

(c)

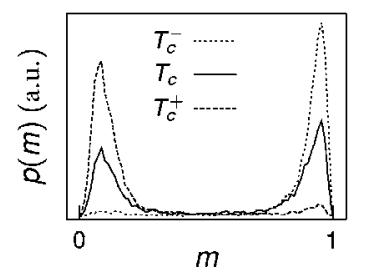

(b)

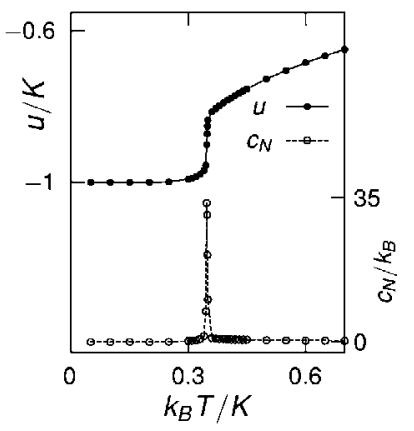

(d)

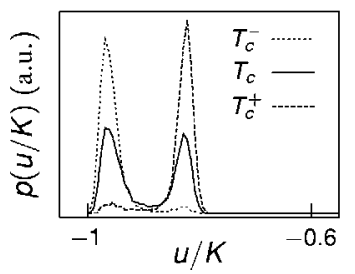

FIG. 15. The herringbone system $(K>0, M / K=-2.1)$. Temperature dependence (a) of the order parameter and its variance and (b) of the energy per dimer and its variance. The distribution functions (c) of the order parameter and (d) energy per dimer. At the phase transition, note the bimodal structure characteristic for a discontinuous phase transition. 


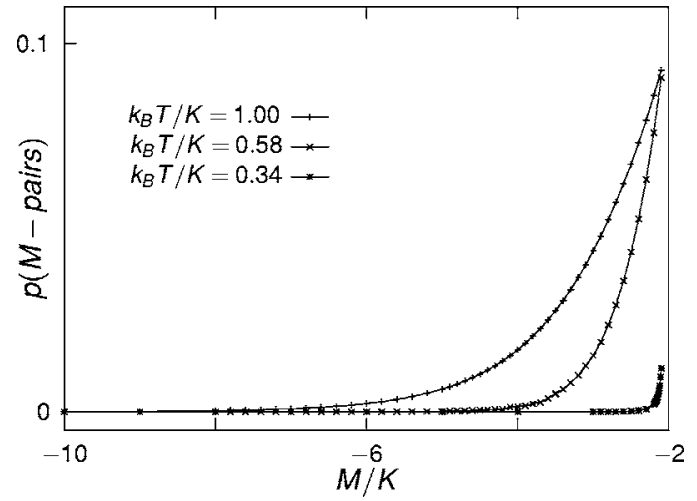

FIG. 16. The probability of finding an $M$ pair $[p(M$ pairs $)$ $\left.=N_{M \text {-pairs }} / 3 N\right]$ in a herringbone system in the HB phase $\left(k_{B} T / K\right.$ $=0.34)$, close to the saturated value of the critical temperature ( $k_{B} T / K=0.58$ and $\left.k_{B} T_{c} / K \approx 0.6\right)$, and well in the $P$ phase $\left(k_{B} T / K\right.$ $=1$ ) as a function of $M / K$. Note that the equilibrium probability for $M$ pairs in the normal $P$ phase (where the probabilities of all dimer orientations are equal, as well as the probabilities of all configurations of dimer pairs) is $1 / 9$.

weakly discontinuous and a continuous transitions. Correspondingly, it is difficult to clearly corroborate the existence of the tricritical point predicted by mean field at $M / K=$ $-7 / 3$, let alone to determine its position. Monitoring the probability distribution of the order parameters suggests that indeed there is a change of the nature of the phase transition in the vicinity of the mean-field prediction.

It is known that quantitatively, the effect of fluctuations decreases the critical temperature obtained within mean field. As it can be clearly seen in Fig. 10, for the HB- $P$ transition this appears to be not the only effect. If in the interval -2 $>M / K \gtrsim-3$ there is still some evidence of the scaling law similar to the one found within the mean-field description, this practically vanishes for $M / K<-3$. Finally, for $M / K<$ -4 the critical temperature "saturates" at a value $k_{B} T_{c} / K$ $\approx 0.6$, i.e., it does not depend any more on the energy ratio $M / K$.

To rationalize this finding, let us recall the expression for the colloidal dimer Hamiltonian (14). The first, $K$ term favors for $K>0$ parallel alignment of dimers. On the other hand, the $M$-term with a large negative value of $M$ strongly suppresses parallel alignment in which the two dimers face each other-to be referred to as an $M$ pair, Fig. 8(b). As a consequence, the probability of finding $M$ pairs in either of the two phases, HB or $P$, is rapidly decreasing with increasing negative values of $M / K$. The number of $M$ pairs as a function of $M / K$ is shown in Fig. 16 for three paths of a constant temperature, viz. in the $\mathrm{HB}$ phase, in the $P$ phase, and at the intermediate temperature $k_{B} T / K=0.58$ for which the system traverses the phase boundary from $\mathrm{HB}$ to $P$ at $M / K \approx-3.7$. For large enough negative $M$ the role of the $M$ term is only to reduce the number of degrees of freedom in the system. Then the corresponding critical behavior depends solely on the relative magnitude of the exchange energy $K$ with respect to the thermal energy $k_{B} T$. In the $\left(k_{B} T /|K|, M /|K|\right)$ phase diagram such a phase boundary is represented by a straight vertical line. Within mean field the reduction of the number (a)

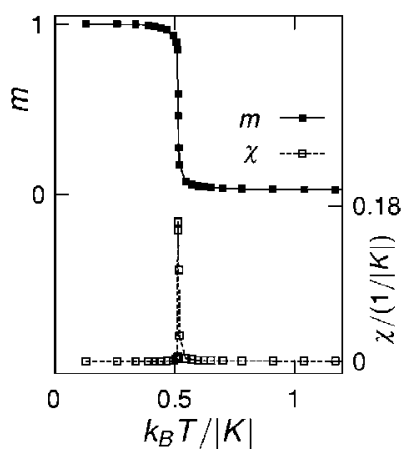

(b)

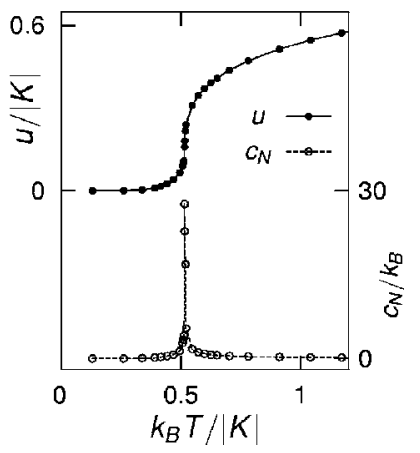

FIG. 17. The antiferromagnetic system $(K<0, M /|K|=0.4)$. Temperature dependence (a) of the order parameter and its variance and (b) of the energy per dimer and its variance.

of degrees of freedom for the configurations of dimer pairs is not properly captured. Thus the role of the $M$ term is as in all the other cases reduced to effectively rescale the exchange energy $K$.

\section{Antiferromagnetic phase}

The ground state of the antiferromagnetically ordered phase occurs in colloidal dimer systems with $K<0, M /|K|$ $<1$. As discussed in Sec. III B, its order can be described by a single order parameter - a sublattice magnetization $m$, where the system is divided into three triangular sublattices.

The AM- $P$ phase transition is discontinuous (see Fig. 17) in the whole range of the stable AM phase. In contradiction to the universal jump of the latent heat and heat capacity determined by the mean-field approach, the MC results show slightly decreasing strength of the discontinuity of the transition when $M /|K|$ is increased towards 1 . At the special point, $M=0$, where the colloidal dimer Hamiltonian reduces to the Potts model, our results match the ones known for the Potts antiferromagnet [30,34]. Applying again the empirical shift of the mean-field analysis to match the antiferromagnetic Potts point gives a satisfactory description of the phase boundary for $M /|K| \geqslant 1$, see Fig. 10. For highly negative $M /|K|$ the critical temperature saturates as is the case of the HB- $P$ transition. The saturation again originates in the suppression of $M$ pairs as already discussed above.

\section{Japanese 6 in 1 system}

For $K<0$, squeezed between the FM and AM phases one observes the new J6/1 phase. Its highly degenerate ground state is stable for $1<M /|K|<5$. The degeneracy of the J6/1 phase originates in the disordered nature of one of its sublattices. A typical snapshot of a ground state in the J6/1 phase is presented in Fig. 18. In addition, a schematic representation and a picture of a chain mail using the Japanese 6 in 1 weaving pattern [35] from which we have borrowed the name are plotted.

The MC simulations corroborate the division of the J6/1 system into four triangular sublattices. Three of them are equivalent and ordered with mutually different preferred dimer orientations for temperatures below the critical one. 
(a)

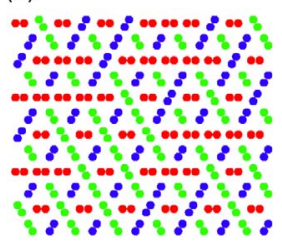

(b)

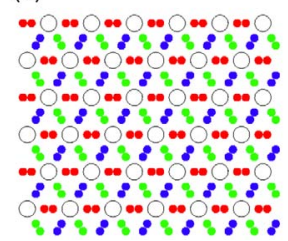

FIG. 18. (Color online) The Japanese 6 in 1 system. (a) A snapshot of one of its ground states. (b) Schematic representation of the J6/1 configuration. Here the circles indicate the degeneracy of the dimer orientation on the disordered sublattice. (c) A picture of chain mail using the Japanese 6 in 1 weaving pattern [35].

The remaining sublattice exhibits no preferred orientation for all temperatures. The magnetization parameter is defined as the average of the three nonzero sublattice magnetizations, $\mathcal{M}=\Sigma_{\Gamma} \mathcal{M}_{\Gamma} / 3$. In the simulation we have monitored both, the magnetization of the ordered as well disordered sublattice. Upon increasing the temperature from the ground state, the order in the ordered sublattices of the J6/1 phase melts and the system exhibits a continuous phase transition to the $P$ phase. The corresponding phase boundary is represented by a curved, nonmonotonic line, see Fig. 10. Close to the critical point the fluctuations of the order parameter are strongly enhanced and the system can "switch" between the four classes of configurations, i.e., characterized by a translation of the disordered sublattice. Graphs of the typical temperature dependences of the sublattice magnetization and energy per dimer are depicted in Fig. 19.

The high residual entropy stabilizes the J6/1 with respect to the neighboring FM or AM phase upon increasing temperature. Correspondingly, the parameter region where the $\mathrm{J} 6 / 1$ phase is stable expands upon heating. For parameters $5<M /|K| \lesssim 5.3$ or $0.8 \lesssim M /|K|<1$ the melting of the FM or $\mathrm{AM}$ proceeds in two steps via a discontinuous structural transition to the J6/1 phase followed by another transition to the paramagnetic state. A typical temperature variation of the energy per dimer and the corresponding heat capacity for the case of a FM-J6/1-P sequence is presented in Fig. 20. The AM-J6/1- $P$ phase sequence is characterized by similar graphs.

(a)

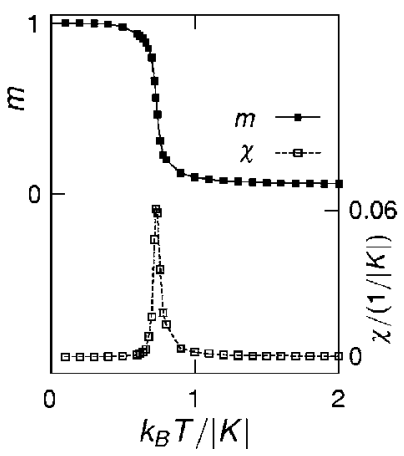

(b)

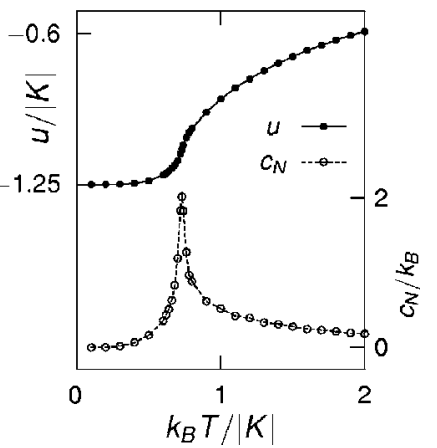

FIG. 19. The Japanese 6 in 1 system $(K<0, M /|K|=3.5)$. Temperature dependence (a) of the (sublattice) order parameter and its variance and (b) of the energy per dimer and its variance (right). Both indicate a continuous nature of the phase transition.

(a)

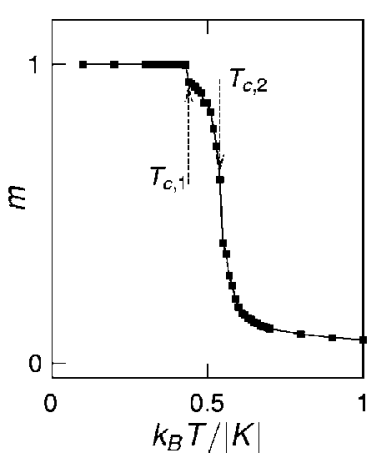

(b)

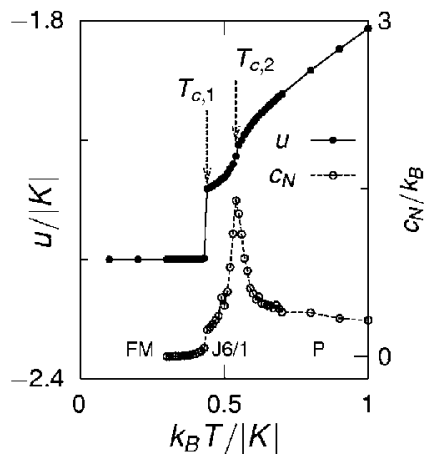

FIG. 20. The system $(K<0, M /|K|=5.2)$ exhibits a sequence of phase transitions FM-J6/1- $P$ upon increasing the temperature. The FM-J6/1 structural transition takes place at $T_{c, 1}$ and is discontinuous whereas there is no jump in the order parameter and energy per dimer at the J6/1-P transition, which takes place at $T_{c, 2}$.

\section{CONCLUSIONS}

We have shown that soft matter colloidal systems exhibit peculiar phase behavior once exposed to external laser fields. The laser-colloid interaction favors configurations where the particles are attracted to the spots of high light intensities. By a subtle balance of the compression of groups of two or three particles at such spots and their mutual repulsion composite objects are formed, such as dimers and trimers. The relevant low-energy degrees of freedom are then the discrete orientational configurations with an excitation spectrum at the thermal scale.

The introduction of an effective Hamiltonian allows us to map the phase behavior to spinlike systems. In particular, we have rationalized the case of trimers on a triangular lattice [22], which has motivated this work, in terms of a twodimensional Ising model. A comparison with experimental data corroborates the approach of using composite objects and validates our theory. The critical properties accompanying the transition are probably hard to study experimentally due to the influence of a finite boundary.

We have suggested a new experimental setup where dimers play the role of the composite objects. It is shown that a rich phase behavior emerges; in terms of a "spin language" a ferromagnetic, an antiferromagnetic, a herringbone, and an exotic Japanese 6 in 1 phase have been determined. We have mapped the phase diagram using a variational mean-field theory and substantiated our findings by Monte Carlo simulations.

It is interesting to note that these colloidal molecular crystals have a counterpart on the atomic scale, viz. adsorption of molecules on atomic surfaces. For instance, nitrogen on graphite $[38,39]$ at suitable densities orders in a herringbone structure similar to the one of colloidal dimers, which lead Sluckin [40] to construct a generalized Potts model closely along the line of reasoning presented here. Allen and Armitstead [41] presented Monte Carlo simulations close to the transition from ferromagnetically ordered nitrogen adsorbates to the experimentally observed herringbone structure. Using solely symmetry arguments, Vollmayr [42] discussed the possible ground states if the interaction between two 
molecules depends on the bond orientation and explored regions of suppressed relaxations in an a priori unfrustrated system.

It appears promising to extend the concept of composite objects to different symmetries of the underlying lattice, e.g., square or rectangular unit cells, and to noninteger filling factors. The corresponding phase diagrams are expected to include a variety of phases and phase transition.

\section{ACKNOWLEDGMENTS}

We thank C. Bechinger for stimulating discussions and acknowledge helpful comments by C. Reichhardt. A.ŠS. acknowledges the support by the Alexander von Humboldt Foundation. T.F. gratefully acknowledges the kind hospitality of the University of Ljubljana where part of this work has been finished.

\section{APPENDIX: RELATION BETWEEN ENERGY SCALES AND EXPERIMENTAL CONTROL PARAMETERS}

In this Appendix we show how the parameters of the trimer Hamiltonian can be expressed in terms of the laser intensity, the screening length of the colloid-colloid interaction, and the effective charge of the colloids. The case of dimers is then a straightforward modification and we merely state the result at the end of this Appendix.

The formation of composite objects, i.e., trimers, as well as their mutual interaction is due to a subtle interplay of the external laser potential and the screened Coulomb colloidcolloid repulsion. The symmetry of the external potential favors equilateral triangles aligned along one of the two distinguished lattice directions. The potential squeezes the constituents of the trimer closer to each other. The forces of the external potential on a trimer are balanced by the internal constituent Coulomb repulsion. To lowest order the effect of the neighboring trimers can be ignored since in the regime of interest the trimers are small compared to the lattice constant. Furthermore, the Coulomb interaction is exponentially suppressed for the case of screening lengths smaller than the lattice constants. The thermal pressure is negligible compared to the binding energy.

To this order of approximation the ground state of the system has a twofold degeneracy per lattice site. Introducing the residual interaction of the trimers, the energy levels are split on a scale comparable with the thermal one.

Let us discuss the interactions of the trimer constituents in detail. First the dielectric contrast at optical frequencies of the colloidal particles with respect to the solvent gives rise to a coupling to the laser intensity. This interaction can be expressed in terms of an effective potential. For the experimental setup under consideration the total electric field consists of a coherent superposition of three laser beams,

$$
\mathbf{E}(\mathbf{r}, t)=\mathbf{E}_{0} e^{-i \omega t}\left[e^{i \mathbf{k}_{1} \cdot \mathbf{r}}+e^{i \mathbf{k}_{2} \cdot \mathbf{r}}+e^{i \mathbf{k}_{3} \cdot \mathbf{r}}\right]+\text { c.c. },
$$

where the respective in-plane projections of wave vectors $\mathbf{k}$ constitute an equilateral triangle. The total intensity consists of a rapidly varying component in time, which averages to zero. The remaining time-independent component gives rise to a constant, which we also discard, and the interesting part, characterized by a spatial modulation,

$$
I(\mathbf{r})=4\left|E_{0}\right|^{2}\left[\cos \left(\mathbf{G}_{1} \cdot \mathbf{r}\right)+\cos \left(\mathbf{G}_{2} \cdot \mathbf{r}\right)+\cos \left(\mathbf{G}_{3} \cdot \mathbf{r}\right)\right],
$$

where $\mathbf{G}_{1}=\mathbf{k}_{2}-\mathbf{k}_{3}, \mathbf{G}_{2}=\mathbf{k}_{3}-\mathbf{k}_{1}$, and $\mathbf{G}_{3}=\mathbf{k}_{1}-\mathbf{k}_{2}$ again form an equilateral triangle.

The periodic potential exhibits the symmetry of a triangular lattice with $\mathbf{G}_{i}$ 's being the wave vectors of the reciprocal lattice. In the following we choose the coordinate system in which

$$
\mathbf{G}_{1}=G(0,-1), \quad \mathbf{G}_{2}=G\left(\frac{\sqrt{3}}{2}, \frac{1}{2}\right), \quad \mathbf{G}_{3}=G\left(-\frac{\sqrt{3}}{2}, \frac{1}{2}\right) .
$$

It is also convenient to introduce the spacial lattice vectors

$$
\mathbf{a}_{1}=a(1,0), \quad \mathbf{a}_{2}=a\left(\frac{1}{2}, \frac{\sqrt{3}}{2}\right), \quad \mathbf{a}_{3}=a\left(-\frac{1}{2}, \frac{\sqrt{3}}{2}\right),
$$

where $a=4 \pi /(G \sqrt{3})$ is the lattice constant of the external potential. In this parametrization and with respect to the lattice sites the relative positions of the colloidal particles within a trimer with orientation $S \in\{+1,-1\}$ are parametrized by the single dimensionless distance parameter $\rho$ :

$$
\mathbf{R}_{1}=S \rho \mathbf{a}_{1}, \quad \mathbf{R}_{2}=S \rho \mathbf{a}_{2}, \quad \mathbf{R}_{3}=S \rho \mathbf{a}_{3} .
$$

The effective interaction of the colloidal particles with the external electric potential is proportional to the laser intensity and the dielectric contrast at optical frequency, i.e.,

$$
\varphi_{D}(\mathbf{r})=-V_{0}\left[I(\mathbf{r}) / 4\left|E_{0}\right|^{2}\right],
$$

where $V_{0}>0$ equals $V_{0}$ used for the parametrization of the external potential in experiment of Ref. [22].

The strong mutual repulsion of the three constituents is mediated via a screened Coulomb interaction

$$
\varphi_{C}(r)=\frac{q^{2}}{r} e^{-\kappa r}
$$

where $\kappa$ is the inverse Debye length, $r$ is the center to center distance, and $q^{2}=\left[\left(Z^{*} e_{0}\right)^{2} /\left(4 \pi \epsilon_{0} \epsilon_{r}\right)\right] e^{\kappa R_{s}} /\left(1+\kappa R_{s}\right)$ with $Z^{*} e_{0}$ being the charge on the surface of a single particle, $\epsilon_{r}$ is the dielectric contrast of water, and $R_{s}$ is the radius of a colloidal particle $[43,44]$. Introducing dimensionless parameters $\rho$ $=r / a$ and $k=\kappa a$ the screened Coulomb repulsion scales as $\varphi_{C}(\rho)=\mathcal{E}_{0} \exp (-k \rho) / \rho$, where $\mathcal{E}_{0}=q^{2} / a$.

The internal potential energy of a single trimer, $E_{C}$ $=\Sigma_{i<j} \varphi_{C}\left(\rho_{i j}\right)$ and $\rho_{i j}$ the distance of two colloids within the trimer,

$$
E_{C} / E_{0}=\frac{\sqrt{3}}{\rho} e^{-k \rho \sqrt{3}},
$$

is competing with the compression of the external dielectric potential, $E_{D}=\Sigma_{i} \varphi_{D}\left(\rho_{i}\right)$ and $\rho_{i}$ the distance of a colloid from the center of mass of the trimer, 


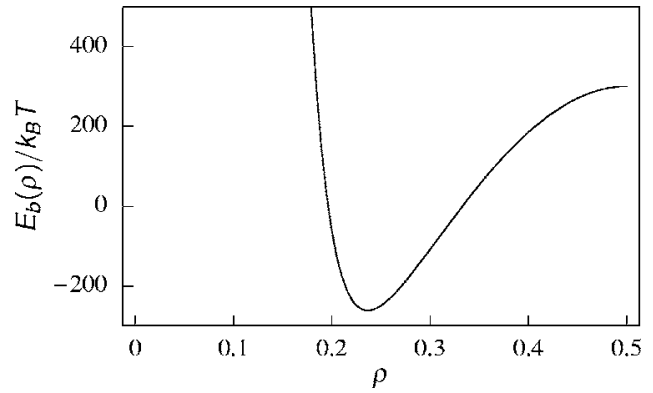

FIG. 21. Dependence of the binding energy of a trimer on its size. The equilibrium trimer extension is determined by the minimum of the binding energy, $\rho \leqslant 1 / 3$.

$$
E_{D} / E_{0}=-3 v_{0}(1+2 \cos 2 \pi \rho),
$$

where $v_{0}=V_{0} / \mathcal{E}_{0}$.

The size of a trimer is determined by minimizing the total potential energy, $E_{C}+E_{H}$, see Fig. 21, with respect to $\rho$. The minimum defines the binding energy $E_{b}$, which for typical experimental parameters exceeds the thermal scale by approximately two orders of magnitude, i.e., $E_{b}$ $\sim 10^{2} k_{B} T$, for $1 / \kappa=570 \mathrm{~nm}, a=11.5 \mu \mathrm{m}, \mathcal{E}_{0}=10^{5} k_{B} T$, and $V_{0}=60-110 k_{B} T$. The separation of scales justifies the idea to treat the assembly of three colloidal particles corresponding to a lattice site as a rigid composite object.

In the picture of composite objects the trimer-trimer interaction enters as residual interaction expected to be much smaller than the binding energy. The trimer-trimer interaction lifts the orientational degeneracy and is the origin of the ordering phenomena under consideration. Since the Coulomb interaction is strongly screened it is sufficient to consider only neighboring lattice sites. For the three different geometrical arrangements of the trimers, see Fig. 2, the interaction energy is determined by the sum of the nine direct colloid-colloid contributions of the rigid triangles,

$$
E_{\mu}=E_{0} \sum_{s, t=1}^{3} \frac{1}{\rho_{\mu}[s, t]} \exp \left(-k \rho_{\mu}[s, t]\right) .
$$

Explicitly, elementary geometrical considerations yield for the tables of the distances in dimensionless units

$$
\begin{gathered}
\rho_{1}=\left[\begin{array}{ccc}
1+2 \rho_{0} & \sqrt{1+\rho_{0}+\rho_{0}^{2}} & \sqrt{1+\rho_{0}+\rho_{0}^{2}} \\
\sqrt{1+\rho_{0}+\rho_{0}^{2}} & \sqrt{1-2 \rho_{0}+4 \rho_{0}^{2}} & 1-\rho_{0} \\
\sqrt{1+\rho_{0}+\rho_{0}^{2}} & 1-\rho_{0} & \sqrt{1-2 \rho_{0}+4 \rho_{0}^{2}}
\end{array}\right], \\
\rho_{2}=\left[\begin{array}{ccc}
1 & \sqrt{1-3 \rho_{0}+3 \rho_{0}^{2}} & \sqrt{1-3 \rho_{0}+3 \rho_{0}^{2}} \\
\sqrt{1+3 \rho_{0}+3 \rho_{0}^{2}} & 1 & \sqrt{1+3 \rho_{0}^{2}} \\
\sqrt{1+3 \rho_{0}+3 \rho_{0}^{2}} & \sqrt{1+3 \rho_{0}^{2}} & 1
\end{array}\right],
\end{gathered}
$$

$$
\rho_{3}=\left[\begin{array}{ccc}
1-2 \rho_{0} & \sqrt{1-\rho_{0}+\rho_{0}^{2}} & \sqrt{1-\rho_{0}+\rho_{0}^{2}} \\
\sqrt{1-\rho_{0}+\rho_{0}^{2}} & \sqrt{1+2 \rho_{0}+4 \rho_{0}^{2}} & 1+\rho_{0} \\
\sqrt{1-\rho_{0}+\rho_{0}^{2}} 1+\rho_{0} & \sqrt{1+2 \rho_{0}+4 \rho_{0}^{2}} &
\end{array}\right],
$$

where $\rho_{0}$ is the equilibrium size of the trimer. For the experimental parameters used above, one obtains $E_{1} \sim 0.01 k_{B} T$, $E_{2} \sim 0.1 k_{B} T, E_{3} \sim 1 k_{B} T$, and thus $J>0$ [see Eq. (4)], which shows that phase transitions are to be expected in the realistic experimental setups.

For the case of dimers the chain of arguments is along the same lines as above, and one finds for the dimer-dimer interactions in the four different geometrical arrangements exhibited in Fig. 8(b),

$$
E_{\mu}=\mathcal{E}_{0} \sum_{s, t=1}^{2} \frac{1}{\rho_{\mu}[s, t]} \exp \left(-k \rho_{\mu}[s, t]\right)
$$

where

$$
\begin{gathered}
\rho_{1}=\left[\begin{array}{cc}
1 & \sqrt{1+2 \rho_{0}+4 \rho_{0}^{2}} \\
\sqrt{1-2 \rho_{0}+4 \rho_{0}^{2}} & 1
\end{array}\right], \\
\rho_{2}=\left[\begin{array}{cc}
1+\rho_{0} & \sqrt{1+3 \rho_{0}^{2}} \\
\sqrt{1+3 \rho_{0}^{2}} & 1-\rho_{0}
\end{array}\right], \\
\rho_{3}=\left[\begin{array}{cc}
\sqrt{1-3 \rho_{0}+3 \rho_{0}^{2}} & \sqrt{1+3 \rho_{0}+3 \rho_{0}^{2}} \\
\sqrt{1-\rho_{0}+\rho_{0}^{2}} & \sqrt{1+\rho_{0}+\rho_{0}^{2}}
\end{array}\right], \\
\rho_{4}=\left[\begin{array}{cc}
1 & 1+2 \rho_{0} \\
1-2 \rho_{0} & 1
\end{array}\right] .
\end{gathered}
$$

For the experimental parameters, as used in the experiment with trimers, one obtains $E_{1} \sim 10^{-2} k_{B} T, E_{2} \sim 10^{-1} k_{B} T, E_{3}$ $\sim 1 k_{B} T, E_{4} \sim 10 k_{B} T$, or equivalently, $K>0$ and $M \ll 0$ which sets the colloidal dimer system in the regime of the herringbone ground state. In order to explore the other regions of the phase diagram one would have to change the colloidcolloid interaction, for example, paramagnetic particles could be used.

Small residual thermal fluctuations of the composite objects, e.g., stretching modes or small oscillations around the minimum of a gross orientational state, can be absorbed in temperature dependent nearest-neighbor interaction energies. For example, one can use a perturbative approach, as has been done for the elastic constants in the case of colloidal suspensions in one-dimensional troughs [17]. 
[1] P. M. Chaikin and T. C. Lubensky, Principles of Condensed Matter Physics (Cambridge University Press, Cambridge, England, 1995).

[2] P. N. Pusey and W. van Megen, Nature (London) 320, 340 (1986).

[3] H. Löwen, Phys. Rep. 237, 249 (1994).

[4] W. B. Russel, D. A. Seville, and W. R. Schowalter, Colloidal Dispersions (Cambridge University Press, Cambridge, England, 1995).

[5] J. Israelachvili, Intermolecular \& Surface Forces (Academic, London, 1985).

[6] C. N. Likos, Phys. Rep. 348, 267 (2001).

[7] M. E. Leunissen, C. G. Christova, A.-P. Hynninen, C. P. Royall, A. I. Campbell, A. Imhof, M. Dijkstra, R. van Roij, and A. van Blaaderen, Nature (London) 437, 235 (2005).

[8] J. M. Kosterlitz and D. J. Thouless, J. Phys. C 6, 1181 (1973).

[9] D. R. Nelson and B. I. Halperin, Phys. Rev. B 19, 2457 (1979).

[10] D. R. Nelson, Defects and Geometry in Condensed Matter Physics (Cambridge University Press, Cambridge, England, 2002).

[11] K. Zahn, R. Lenke, and G. Maret, Phys. Rev. Lett. 82, 2721 (1999).

[12] A. Chowdhury, B. J. Ackerson, and N. A. Clark, Phys. Rev. Lett. 55, 833 (1985).

[13] Q.-H. Wei, C. Bechinger, D. Rudhardt, and P. Leiderer, Phys. Rev. Lett. 81, 2606 (1998).

[14] C. Bechinger, M. Brunner, and P. Leiderer, Phys. Rev. Lett. 86, 930 (2001).

[15] K. Mangold, P. Leiderer, and C. Bechinger, Phys. Rev. Lett. 90, 158302 (2003).

[16] K. H. Lin, J. C. Crocker, V. Prasad, A. Schofield, D. A. Weitz, T. C. Lubensky, and A. G. Yodh, Phys. Rev. Lett. 85, 1770 (2000).

[17] E. Frey, D. R. Nelson, and L. Radzihovsky, Phys. Rev. Lett. 83, 2977 (1999).

[18] L. Radzihovsky, E. Frey, and D. R. Nelson, Phys. Rev. E 63, 031503 (2001).

[19] Y. Enomoto, M. Sawa, and H. Itamoto, Physica A 345, 547 (2005).

[20] C. Reichhardt and C. J. Olson Reichhardt, Phys. Rev. E 72, 032401 (2005).

[21] J. Baumgartl, M. Brunner, and C. Bechinger, Phys. Rev. Lett. 93, 168301 (2004).

[22] M. Brunner and C. Bechinger, Phys. Rev. Lett. 88, 248302 (2002).

[23] C. Reichhardt and C. J. Olson, Phys. Rev. Lett. 88, 248301
(2002)

[24] R. Agra, F. van Wijland, and E. Trizac, Phys. Rev. Lett. 93, 018304 (2004).

[25] C. Reichhardt and C. J. Olson Reichhardt, Phys. Rev. E 71, 062403 (2005).

[26] A. Šarlah, T. Franosch, and E. Frey, Phys. Rev. Lett. 95, 088302 (2005).

[27] R. J. Baxter, Exactly Solved Models in Statistical Mechanics (Academic, London, 1989).

[28] C. Bechinger and E. Frey, J. Phys.: Condens. Matter 13, R321 (2001).

[29] R. J. Baxter, J. Phys. C 6, L445 (1973).

[30] F. Y. Wu, Rev. Mod. Phys. 54, 235 (1982), and the references therein.

[31] K. Binder, J. Stat. Phys. 24, 69 (1981).

[32] M. Schick and R. B. Griffiths, J. Phys. A 10, 2123 (1977).

[33] I. G. Enting and F. Y. Wu, J. Stat. Phys. 28, 351 (1982).

[34] Y. Saito, J. Phys. A 15, 1885 (1982).

[35] Matthew Durnford and http://www.mailleartisans.org

[36] N. Metropolis, A. W. Rosenbluth, M. N. Rosenbluth, A. H. Teller, and E. Teller, J. Chem. Phys. 21, 1087 (1953).

[37] K. Binder and D. W. Heermann, Monte Carlo Simulations in Statistical Physics, 4th ed. (Springer-Verlag, Berlin, 2002).

[38] T. T. Chung and J. G. Dash, Surf. Sci. 66, 559 (1977).

[39] R. D. Diehl, M. F. Toney, and S. C. Fain, Phys. Rev. Lett. 48, 177 (1982).

[40] T. J. Sluckin, J. Phys. A 21, 1415 (1988).

[41] M. P. Allen and D. J. Tildesley, Computer Simulation of Liquids (Clarendon, Oxford, 1989).

[42] H. Vollmayr, Phys. Rev. B 46, 733 (1992).

[43] B. V. Derjaguin and L. Landau, Acta Physicochim. URSS 14, 633 (1941).

[44] E. J. W. Vervey and J. T. G. Overbeek, Theory of the Stability of Lyophobic Colloids (Elsevier, Amsterdam, 1948).

[45] G. Grimmett and D. R. Stirzaker, Probability and Random Processes (Clarendon, Oxford, 1995).

[46] Within Monte Carlo simulations the term Monte Carlo cycle denotes the number of attempted moves one should perform to assure that on average each spin is selected once. Although the problem of finding the appropriate number (known as Coupon collector's problem) is exactly solved, see, e.g., [45], the factor $\ln N$ is often omitted in the physics literature. In simulations, this is then "corrected" by either analyzing a huge number of configurations or by sampling only configurations being separated a certain number of cycles. In the vicinity of phase transitions with pronounced correlations this has to be done anyway. 